\title{
Mappings of finite order and dimension theory
}

\author{
By Keiô Nagami \\ (Received February 15, 1960)
}

\section{Contents}

$\S 1$. Introduction.

$\S 2$. Zero-dimensional spaces.

$\S 3$. Fundamental relations in dimension theory for metric spaces.

$\S 4$. Open mappings whose order are point-wise finite.

$\S 5$. Cuts.

$\S 6$. W. Hurewicz's problem.

$\S 7$. Characterization of dimension by means of a sequence of coverings.

$\S 8$. Subordinate metric spaces.

§. Dimension-preserving completion.

$\S 10$. Length of coverings.

$\S 11$. Characterization of dimension of non-metrizable spaces.

\section{§1. Introduction}

Among several functions called dimension the following two definitions of dimension are surely the most important ones for non-separable spaces. One is the covering dimension due to $\mathrm{H}$. Lebesgue defined as follows.

Definition 1.1. Let $R$ be a normal space. $R$ has the covering demension $\leqq n^{1)}$, $\operatorname{dim} R \leqq n$, if the following condition is satisfied.

(1.1) For any finite open covering $\mathbb{B}$ there exists an open covering $\mathfrak{S}$ with order $\mathfrak{S}^{2)} \leqq n+1$ such that $\mathfrak{S}$ refines ${ }^{(3 .}{ }^{3)} \quad$ We call $\operatorname{dim} R=n$ if $\operatorname{dim} R \leqq n$ is true and $\operatorname{dim} R \leqq n-1$ is false. When $R=\phi=$ the empty set, we call $\operatorname{dim} \phi=-1$. When there is no such $n$ as $\operatorname{dim} R \leqq n$, we call $\operatorname{dim} R=\infty$.

Another is the large inductive dimension due to E. Čech defined as follows.

Definition 1.2. Let $R$ be a normal space. The empty set $\phi$ has the large inductive dimension -1 , Ind $\phi=-1$. $R$ has the large inductive dimension $\leqq n$, Ind $R \leqq n$, if

(1.2) For any pair of a closed set $F$ and an open set $G$ with $F \subset G$ there exists an open set $U$ with $F \subset U \subset G$ such that Ind $(\bar{U}-U) \leqq n-1$. We call Ind $R=n$ if Ind $R \leqq n$ is true and Ind $R \leqq n-1$ is false. When there is no such $n$, we call Ind $R=\infty$.

Our notion "Ind" is the same as "ind dim" of K. Morita [16] or " $d_{2}$ " of W. Hurewicz and H. Wallman [8, Appendix].

1) Throughout this paper $n$ denotes an integer with $n \geqq-1$.

2) order $\mathfrak{H}$ denotes the order of $\mathfrak{H}$, i.e., the largest integer $m$ such that there are $m$ elements of $\mathscr{S}$ which have a non-empty intersection. When there does not exist such $m$, order $\mathfrak{S}=\infty$.

3) Henceforth $\mathfrak{S}>\mathfrak{F}$ denotes the fact that $\mathfrak{H}$ refines $\mathfrak{H}$. When every element of $\mathfrak{F}$ is contained in some element of $\mathbb{S}$, we say that $\mathfrak{H}$ refines $\mathbb{S}$ or $\mathfrak{S}$ is a refinement of $\mathbb{S}$. 
It is to be noted that the condition (1.1) used in the definition of the covering dimension can be replaced with any one of the following conditions (cf. C. H. Dowker [3] or K. Morita [14; 15]).

(1.3) Any locally finite ${ }^{4)}$ open covering $\$ \$$ can be refined by an open covering $\mathfrak{g}$ of order $\leqq n+1$.

(1.4) Any locally finite open covering $\mathbb{B}=\left\{G_{\alpha} ; \alpha \in A\right\}$ can be refined by an open covering $\mathfrak{S}=\left\{H_{\alpha} ; \alpha \in A\right\}$ of order $\leqq n+1$ such that $H_{\alpha} \subset G_{\alpha}$ for every $\alpha \in A$.

Although we were able to set up a definition of dimension for non-separable spaces, yet it was not possible to establish a theory of dimension for such general spaces (cf. W. Hurewicz and H. Wallman [8, Appendix]). Thus the dimension theory had been obliged to confine itself to only separable metric spaces. Recently the dimension theory for general metric spaces has been established by M. Katětov [10] and by K. Morita [16] independently with different proofs. They have shown the equivalence of the convering dimension and the large inductive dimension for general metric spaces and extended the fundamental theorems of dimension theory to such spaces.

The principal contribution of this paper is to furnish the third systematic development of dimension theory, other than Katětov and Morita's, for general metric spaces. The full use of closed mappings of finite order will simplify greatly our theory, where the order of mappings is defined as follows.

Definition 1.3. Let $f$ be a mapping of a topological space $R$ into another topological space $S$ and $x$ be a point of $S$. The order of $f$ at $x$, order $(x, f)$, is $\left|f^{-1}(x)\right|$ (=the cardinal number of the set $f^{-1}(x)$ ). The order of $f$, order $f$, is $\sup \left\{\left|f^{-1}(x)\right| ; x \in S\right\}$. When order $(x, f)$ is finite at every point $x \in S$, we call that the order of $f$ is point-wise finite.

A characterization of zero-dimensional metric spaces which is essentially the same as in Katétov [10] and in Morita [16] is given in $\S 2$ for the sake of completeness and of preparation for the next paragraph.

In $\S 3$ the fundamental six propositions which characterize $n$-dimensionality of metric spaces will be proved to be equivalent to each other (Theorem 3.2) with the aid of Theorem 3.1 which asserts that a closed continuous image $S$ of a 0 -dimensional metric space $R$ under a mapping $f$ such that $\operatorname{order}(y, f)=k(<\infty)$ for any point $y \in S$ has dimension 0 . Theorem 3.1 can be generalized for the case of $n$-dimension (Theorem 3.7 $)^{5}$. Although the proof of Theorem 3.7 is more simple, with the aid of other results, than that of Theorem 3.1, rather complicated proof of Theorem 3.1 is self-contained and of some interest by itself. Two sorts of covering theorems will also be stated in Theorems 3.5 and 3.6. These theorems have been proved independently by Prof. Morita and are closely related with works of Morita [16, $\$ 9 ; 18]$ and of Katétov [11, Sect. 2].

$\S 4$ deals with an open mapping and dimension. It will be shown that an open continuous mapping whose order is point-wise finite cannot increase dimension for some class of non-metrizable spaces.

$\S 5$ gives a definition of the cut and a proof of the existence of it. Some

4) A collection $\Re$ of subsets of $R$ is called locally finite if for every point $x \in R$ there is a neighborhood of $x$ which meets only a finite number of elements of $\AA$.

5) Theorem 3.7 is proved by J. Suzuki [28] independently. 
properties of it is also studied. The cut and Theorem 3.5 will be the main instrument to solve the problem dealt with in the next paragraph.

In $\S 6$ we construct a metric space $T$ with $\operatorname{dim} T=m$ for an arbitrary metric space $R$ with $\operatorname{dim} R=n, 0<n(<\infty)$, and for an arbitrary integer $m$ with $0 \leqq m<n$ such that there exists a closed continuous mapping $\pi_{0}$ of $T$ onto $R$ with order $\pi_{0}=$ $n-m+1$. This is an answer for W. Hurewicz's problem for general metric spaces.

The following is the well-known P. Alexandroff's approximation theorem (cf. Hurewicz and Wallman [8, p. 72]).

In order that a compact metric space $R$ has dimension $\leqq n$ it is necessary and sufficient that for every positive $\varepsilon$ there is an $\varepsilon$-mapping of $R$ into a finite polytope of dimension $\leqq n$. form.

It is also well-known that this theorem can be expressed in the following

In order that a compact metric space $R$ has dimension $\leqq n$ it is necessary and sufficient that there exists a sequence of open coverings $\mathfrak{H}_{i}, i=1,2, \cdots$, of $R$ which satisfies the following conditions.

(1.5) order $\mathfrak{U}_{i} \leqq n+1$ for every $i$.

(1.6) $\left.\operatorname{mesh} \mathfrak{H}_{i}{ }^{6}\right) \rightarrow 0$ as $i \rightarrow \infty$.

Whether this proposition is valid for non-compact (even separable) metric spaces is a famous Alexandroff's problem. Recently this problem has been solved in the negative by K. Sitnikov [27] who has constructed an example of a metric space $R$ with $\operatorname{dim} R=2$ whose metric dimension ${ }^{7)}$ is 1 . More recently M. Katětov [12] has proved that if $R$ is a metric space, then met $\operatorname{dim} R \leqq$ $\operatorname{dim} R \leqq 2 \operatorname{met} \operatorname{dim} R$. The reason why the existence of a sequence satisfying the above conditions characterizes $n$-dimensionality of a compact metric space is as follows: Let $\mathfrak{H}_{i}, i=1,2, \cdots$, be such a sequence of a compact metric space $R$. Let $\mathfrak{F}_{i}$ be a finite subcovering of $\mathfrak{H}_{i}$. Then there exists a subsequence $\mathfrak{F}_{i^{\prime}}$, $i=1,2, \cdots$, such that $\left.\widetilde{F}_{i^{\prime}}>\overline{\mathfrak{F}}_{(i+1)}, 8\right)$, for every $i$. Then the subsequence $\widetilde{F}_{i^{\prime}}, i=$ $1,2, \cdots$, satisfies the condition (B) of $\S 3$, which proves $\operatorname{dim} R \leqq n$ by Theorem 3.2. By these observations we know that the equivalence of $(A)$ and $(B)$ of Theorem 3.2 is a generalization of Alexandroff's theorem cited above. Besides ours characterizations of dimension by means of a sequence of coverings have already been given by J. Nagata [24, Theorem 3] and by C. H. Dowker and W. Hurewicz [6]. In $\S 7$ we shall give weaker conditions imposed on a sequence which characterize dimension than those in (B) of $\S 3$, J. Nagata [24, Theorem 3] and C. H. Dowker and W. Hurewicz [6] (Theorems 7.2 and 7.5).

In $\S 8$ we observe the dimension of subordinate metric spaces (as for the definition see Definition 8.1) as an application of $\S 7$.

The existence of a dimension-preserving completion of a metric space has been proved by Katětov [10, Theorem 3.10] and by Morita [16, Theorem 5.6] independently. In $\S 9$ we shall execute to construct a dimension-preserving

6) The mesh of a collection $\mathfrak{u}$ of subsets of $R$ is the largest of the diameters of the elements of $\mathfrak{u}$.

7) When there is a sequence of open coverings $\mathfrak{u}_{i}, i=1,2, \cdots$, of a metric space $R$ satisfying (1.5) and (1.6), we call that $R$ has the metric dimension $\leqq n$, met $\operatorname{dim} R \leqq n$. When met $\operatorname{dim} R \leqq n$ is true and met $\operatorname{dim} R \leqq n-1$ is false, we call that met $\operatorname{dim} R=n$.

8) $\overline{\mathfrak{U}}$ denotes $\{\bar{U} ; U \in \mathfrak{U}\}$, where $\mathfrak{u}$ is a collection of subsets of a topological space. 
completion of a metric space with the aid of our characterization theorem in $\S 7$ (Theorem 9.1). From our construction we can know that a completion of a metrizable space with respect to J. Nagata's metric (cf. Definition 9.3) is a dimension-preserving one (Theorem 9.4).

In $\S 10$ our concern is devoted to the notion "length of a multiplicative covering" which is due to P. Alexandroff and A. Kolmogoroff [1] and has been recently applied to the characterization of dimension of metric spaces by $\mathrm{J}$. Nagata [24, Theorem 4]. Our Theorem 10.2 gives a weaker condition than those in $(\mathrm{B})$ of $\S 3$.

Finally $\S 11$ gives a characterization of the covering dimension of normal spaces by means of the length of coverings.

The content of this paper includes a development in detail of our brief notes $[21 ; 22 ; 23]$.

I have been stimulated interest in dimension theory by Professor K. Morita who has been kind enough to give instructive advices and constant encouragement to me for many years. During the preparation of this paper he has given me many valuable suggestions. I express here my hearty thanks to Professor Morita.

\section{$\S 2$. Zero-dimensional spaces}

Theorem 2.1. A topological space $R$ is a metric space with $\operatorname{dim} R \leqq 0$ if and only if $R$ is a dense subset of an inverse limiting space of a sequence of discrete spaces.

Proof. To prove the necessity let $R$ be a metric space with $\operatorname{dim} R \leqq 0$. Then for every positive integer $i$ there exists an open covering $\mathfrak{H}_{i}$ with order $\mathfrak{H}_{i} \leqq 1$ and mesh $\mathfrak{H}_{i}<2^{-i}$. When $R \neq \phi$ i.e. $\operatorname{dim} R=0$, we assume with no loss of generality that each element of $\mathfrak{H}_{i}$ is not empty. Let $\mathfrak{B}_{i}=\bigcap_{j \leqq i} \mathfrak{H}_{j}{ }^{9)}$; then $\mathfrak{B}_{i}=\left\{V_{\alpha} ; \alpha \in A_{i}\right\}$ is an open covering of $R$ such that i) order $\mathfrak{B}_{i} \leqq 1$, ii) mesh $\mathfrak{B}_{i}<2^{-i}$, iii) $\mathfrak{B}_{i+1}$ refines $\mathfrak{B}_{i}$. Define $f_{i+1, i}: A_{i+1} \rightarrow A_{i}$ in such a way that $f_{i+1, i}(\alpha)=\beta$ yields $V_{\alpha} \subset V_{\beta}$. Since $\mathfrak{B}_{i+1}$ is a refinement of $\mathfrak{B}_{i}, f_{i+1, i}$ can be defined on $A_{i+1}$. Consider $A_{i}$ as a topological space with the discrete topology. Let $A$ be an inverse limiting space of a system $\left\{A_{i}, f_{i+1, i}\right\}, A=\lim \left\{A_{i}, f_{i+1, i}\right\}$. Let $A_{0}$ be a subspace of $A$ consisting of points $\left(\alpha_{1}, \alpha_{2}, \cdots\right) \in A$ such that $\bigcap_{i=1}^{\infty} V_{\alpha_{i}} \neq \phi$. When $\bigcap_{i=1}^{\infty} V_{\alpha_{i}} \neq \phi$, then it consists of a single point. Let $\varphi$ be a mapping of $A_{0}$ into $R$ such that $\varphi(a)=\bigcap_{i=1}^{\infty} V_{\pi_{\boldsymbol{i}}(a)}$, where $\pi_{i}$ is the projection of $A_{0}$ onto $A_{i}$.

Let $x$ be an arbitrary point of $R$; then for every $i$ there exists one and only one element $V_{\alpha_{i}}$ of $\mathfrak{B}_{i}$ such that $x \in V_{\alpha_{i}}$. Since $V_{\alpha_{i+1}} \subset V_{\alpha_{i}}$ for every $i, a=$ $\left(\alpha_{1}, \alpha_{2}, \cdots\right)$ is a point of $A_{0}$ and $\varphi(a)=x$. Hence $\varphi$ is onto.

Let $a$ and $b$ be points of $A_{0}$ with $a \neq b$; then there exists $i$ such that $\pi_{i}(a) \neq \pi_{i}(b)$. Since $V_{\pi_{i}(a)} \cap V_{\pi_{i}(b)}=\phi$, we get $\varphi(a) \neq \varphi(b)$. Thus $\varphi$ is one-to-one.

Let $a$ be an arbitrary point of $A_{0}$; then $\varphi\left(\pi_{i}^{-1}\left(\pi_{i}(a)\right)\right)=V_{\pi_{i}(a)}$ for every $i$, which proves the continuity of $\varphi$ and $\varphi^{-1}$. Therefore $\varphi$ is a homeomorphism of $A_{0}$ onto $R$. Since $\pi_{i}\left(A_{0}\right)=A_{i}$ for every $i, A_{0}$ is a dense subset of $A$.

To prove the sufficiency let $R$ be a dense subset of an inverse limiting space $\lim \left\{A_{i}, f_{i+1, i}\right\}$ of discrete spaces $A_{i}$ and $\mathbb{B}_{S}=\left\{G_{1}, \cdots, G_{m}\right\}$ an arbitrary

9) $\bigcap_{j \leqq i} \mathfrak{u}_{j}$ denotes the aggregate of sets of type $\bigcap_{j \leqq i}\left\{U_{j} ; U_{j} \in \mathfrak{U}_{j}\right\} \neq \phi$. 
finite open covering of $R$. Let $\pi_{i}$ be the projection of $R$ onto $A_{i}$. Let $B_{i j}=$ $\left\{\alpha ; \alpha \in A_{i}-\bigcup\left\{f_{i k}^{-1}\left(B_{k l}\right) ; l=1, \cdots, m, k=1, \cdots, i-1\right\}, \pi_{i}^{-1}(\alpha) \subset G_{j}\right.$ and $\pi_{i}^{-1}(\alpha) \not \subset G_{k}$ for $k=1, \cdots, j-1\}^{10)}$. Then it can easily be seen that $\left\{U_{i j}=\bigcup\left\{\pi_{i}(\alpha) ; \alpha \in B_{i j}\right\}\right.$; $j=1, \cdots, m, i=1,2, \cdots\}$ is a covering of $R$ consisting of mutually disjoint open sets. Moreover $U_{j}=\bigcup_{i=1}^{\infty} U_{i j}$ is included in $G_{j}$ for $j=1, \cdots, m$. Hence $\mathfrak{u}=$ $\left\{U_{j} ; j=1, \cdots, m\right\}$ is an open covering of $R$ with order $\mathfrak{U} \leqq 1$ which refines $(\xi 5$. Therefore $\operatorname{dim} R \leqq 0$ and the theorem is completely proved.

\section{§3. Fundamental relations in dimension theory for metric spaces}

Throughout this paragraph $R$ is a metric space. The following propositions which are fundamental in dimension theory for metric spaces are proved to be equivalent in such a way as $(\mathrm{A}) \rightarrow(\mathrm{B}) \rightarrow(\mathrm{C}) \rightarrow(\mathrm{D}) \rightarrow(\mathrm{E}) \rightarrow(\mathrm{A})$.

(A) $\operatorname{dim} R \leqq n$.

(B) There exists a sequence of locally finite coverings $\widetilde{F}_{i}=\left\{F_{\alpha} ; \alpha \in A_{i}\right\}, i=$ $1,2, \cdots$, of $R$ such that

(3.1) $\overline{\widetilde{\mho}}_{i+1}$ refines $\mathfrak{\mho}_{i}$ for every $i$,

(3.2) order $\mathfrak{\mho}_{i} \leqq n+1$ for every $i$,

(3.3) for any neighborhood $U$ of any point $x$ of $R$ there exists $i$ with $\operatorname{Star}\left(x, \widetilde{\mho}_{i}\right)^{11)} \subset U$.

(C) There exist a metric space $A_{0}$ with $\operatorname{dim} A_{0} \leqq 0$ and a closed continuous mapping $f$ of $A_{0}$ onto $R$ with order $f \leqq n+1$.

(D) $R$ is the sum of $n+1$ subspaces $R_{i}, i=1, \cdots, n+1$, with $\operatorname{dim} R_{i} \leqq 0$.

(E) Ind $R \leqq n$.

(A) implies (B). Let $\mathfrak{H}_{1}=\left\{U_{\alpha} ; \alpha \in A_{1}\right\}$ be a locally finite open covering of $R$ of order $\leqq n+1$ such that the diameter of each $U_{\alpha}<1$. Since a locally finite open covering is shrinkable ${ }^{12)}$, there exist a closed covering $\widetilde{\mho}_{1}=\left\{F_{\alpha} ; \alpha \in A_{1}\right\}$ and an open covering $\mathfrak{B}_{1}=\left\{V_{\alpha} ; \alpha \in A_{1}\right\}$ such that $U_{\alpha} \supset F_{\alpha} \supset V_{\alpha}$ for every $\alpha \in A_{1}$. Let $\mathfrak{H}_{2}=\left\{U_{\alpha} ; \alpha \in A_{2}\right\}$ be a locally finite open covering of order $\leqq n+1$ such that the diameter of each $U_{\alpha}\left(\alpha \in A_{2}\right)<1 / 2$ and $\mathfrak{H}_{2}$ refines $\mathfrak{B}_{1}$. Let $\mathfrak{F}_{2}=\left\{F_{\alpha} ; \alpha \in A_{2}\right\}$ and $\mathfrak{V}_{2}=\left\{V_{\alpha} ; \alpha \in A_{2}\right\}$ be respectively a closed covering and an open covering of $R$ such that $U_{\alpha} \supset F_{\alpha} \supset V_{\alpha}$ for every $\alpha \in A_{2}$. Repeating this procedure we get a sequence of closed coverings $\widetilde{\mho}_{i}=\left\{F_{\alpha} ; \alpha \in A_{i}\right\}, i=1,2, \cdots$, which satisfies the conditions (3.1), (3.2) and (3.3).

(B) implies (C). Let $\widetilde{r}_{i}=\left\{F_{\alpha} ; \alpha \in A_{i}\right\}, i=1,2, \cdots$, be locally finite coverings of $R$ which satisfy the conditions (3.1), (3.2) and (3.3). For every $i$ let us define a single-valued mapping $f_{i+1, i}$ of $A_{i+1}$ into $A_{i}$ in such a way that $f_{i+1, i}(\alpha)=\boldsymbol{\beta}$ yields $\bar{F}_{\alpha} \subset F_{\beta}$. For $i$ and $j$ with $i>j$ let $f_{i j}=f_{j+1, j} \cdots f_{i-1, i-2} f_{i, i-1}$. Let $A$ be an inverse limiting space of a system $\left\{A_{i}, f_{i j}\right\}, A=\lim \left\{A_{i}, f_{i j}\right\}$ where $A_{i}$ are topological spaces with discrete topology. Let $A_{0}$ be a subspace of $A$ consisting of points $\left(\alpha_{1}, \alpha_{2}, \cdots\right) \in A$ such that $\bigcap_{i=1}^{\infty} F_{\alpha_{i}} \neq \phi$. When $\bigcap_{i=1}^{\infty} F_{\alpha_{i}} \neq \phi$, then it consists of a single point by (3.3). Let $f$ be a mapping of $A_{0}$ into $R$ such that $f(a)=\bigcap_{i=1}^{\infty} F_{\pi_{i}(a)}$, where $\pi_{i}$ is the projection of $A_{0}$ into $A_{i}$.

10) $f_{i k}=f_{k+1, k} \cdots f_{i-1, i-2} f_{i, i-1}$.

11) $\operatorname{Star}\left(x, \mathfrak{\mho}_{i}\right)=\bigcup\left\{F_{\alpha} ; x \in F_{\alpha} \in \mathfrak{\mho}_{i}\right\}$.

12) An open covering $\left\{U_{\alpha} ; \alpha \in A\right\}$ is called shrinkable if there exists a close covering $\left\{F_{\alpha} ; \alpha \in A\right\}$ such that $U_{\alpha} \supset F_{\alpha}$ for every $\alpha \in A$. A point-finite open covering of a normal space is shrinkable (cf. [13]). 
Let $x$ be an arbitrary point of $R$. Since $\mathfrak{F}_{i}$ is locally finite, $B_{i}=\left\{\alpha ; x \in F_{\alpha} \in \mathfrak{F}_{i}\right\}$ $\left(\subset A_{i}\right)$ is a non-empty finite set of indices. Since $f_{i+1, i}\left(B_{i+1}\right) \subset B_{i}$ for every $i$, $\left\{B_{i}, f_{i+1, i} \mid B_{i+1}\right\}$ forms an inverse system. Consider $B=\lim \left\{B_{i}, f_{i+1, i} \mid B_{i+1}\right\}$ and then evidently $B \subset A$. Since an inverse limiting space of a sequence of nonempty compact spaces is not empty, $B \neq \phi$. Let $\left(\alpha_{1}, \alpha_{2}, \cdots\right)$ be a point of $B$; then $\bigcap_{i=1}^{\infty} F_{\alpha_{i}}=x$ and hence $f(a)=x$, which proves that $f$ is onto.

Let $a$ be an arbitrary point of $A_{0}$ and $U$ an arbitrary neighborhood of $f(a)$; then there exists $i$ with $\operatorname{Star}\left(f(a), \widetilde{\mho}_{i}\right) \subset U$ by (3.3). Since $f(a) \in F_{\pi_{\boldsymbol{\pi}_{i}}(a)}$ and $f\left(\pi_{i}^{-1}\left(\pi_{i}(a)\right)\right) \subset F_{\pi_{i}(a)}$, we get $f\left(\pi_{i}^{-1}\left(\pi_{i}(a)\right)\right) \subset U$, which proves the continuity of $f$.

To prove order $f \leqq n+1$ assume that order $f>n+1$ and then there exists a point $x$ of $R$ with $\operatorname{order}(x, f)>n+1$. Let $a_{i}, i=1, \cdots, n+2$, be mutually different points of $f^{-1}(x)$; then there exists $j$ such that $\pi_{j}\left(a_{i}\right), i=1, \cdots, n+2$, are mutually different indices of $A_{j}$. Since $x=f\left(a_{i}\right) \in f\left(\pi_{j}^{-1}\left(\pi_{j}\left(a_{i}\right)\right)\right) \subset F_{\pi_{j}\left(a_{i}\right)}$ for $i=1, \cdots, n+2$, order $\widetilde{\mho}_{j}>n+1$ which contradicts to (3.2). Thus we get order $f \leqq n+1$.

To show the closedness of $f$ let $F$ be an arbitrary closed set of $A_{0}$. Then $f(F)=\bigcup\left\{f\left(F \cap \pi_{1}^{-1}(\alpha)\right) ; \alpha \in \pi_{1}\left(A_{0}\right)\right\}$. Since $f\left(\pi_{1}^{-1}(\alpha)\right) \subset F_{\alpha}$ for any $\alpha \in \pi_{1}\left(A_{0}\right)$ and $\mathfrak{\mho}_{1}=\left\{F_{\alpha} ; \alpha \in A_{1}\right\}$ is locally finite, we get $\overline{f(F)}=\bigcup\left\{\overline{f\left(F \cap \pi_{1}^{-1}(\alpha)\right)} ; \alpha \in \pi_{1}\left(A_{0}\right)\right\}$. Let $x_{0}$ be a given point of $\overline{f(F)}$ and then there exists $\alpha_{1} \in \pi_{1}\left(A_{0}\right)$ such that $x_{0} \in \overline{f\left(F \cap \pi_{1}^{-1}\left(\alpha_{1}\right)\right)}$. By the inductive process we can find an index $\alpha_{i} \in \pi_{i}\left(A_{0}\right)$ for $i=1,2, \cdots$, such that $x_{0} \in \overline{f\left(F \cap \pi_{1}^{-1}\left(\alpha_{1}\right) \cap \cdots \cap \pi_{i}^{-1}\left(\alpha_{i}\right)\right)}$. Since $\pi_{i}^{-1}\left(\alpha_{i}\right) \cap \pi_{i+1}^{-1}\left(\alpha_{i+1}\right) \neq \phi$ for any $i$, it holds that $f_{i+1, i}\left(\alpha_{i+1}\right)=\alpha_{i}$ for any $i$. Therefore $a=\left(\alpha_{1}, \alpha_{2}, \cdots\right)$ is a point of $A$. Since $f\left(\pi_{i+1}^{-1}\left(\alpha_{i+1}\right)\right) \subset F_{\alpha_{i+1}}$ and $\bar{F}_{\alpha_{i+1}} \subset F_{\alpha_{i}}$ for any $i$,

$$
\left.x_{0} \in \bigcap_{i=1}^{\infty} \overline{f\left(F \cap \pi_{1}^{-1}\left(\alpha_{1}\right) \cap \cdots \cap \pi_{i}^{-1}\left(\alpha_{i}\right)\right)} \subset \bigcap_{i=1}^{\infty} \overline{f\left(\pi_{i}^{-1}\left(\alpha_{i}\right)\right.}\right) \subset \bigcap_{i=1}^{\infty} \bar{F}_{\alpha_{i}} \subset \bigcap_{i=2}^{\infty} \bar{F}_{\alpha_{i}} \subset \bigcap_{i=1}^{\infty} F_{\alpha_{i}} .
$$

Since $\bigcap_{i=1}^{\infty} F_{\alpha_{i}}$ is either the empty set or a single point, we get $x_{0}=\bigcap_{i=1}^{\infty} F_{\alpha_{i}}$. Thus we can know that $a$ is a point of $A_{0}$ and $f(a)=x_{0}$. On the other hand $\left\{\pi_{i}^{-1}\left(\alpha_{i}\right) ; i=1,2, \cdots\right\}$ is a complete system of neighborhoods of $a$ and $F \cap \pi_{i}^{-1}\left(\alpha_{i}\right) \neq \phi$ for any $i$. Therefore $a \in \bar{F}=F$ and $x_{0}=f(a) \in f(F)$, which shows the closedness of $f(F)$. Thus the closedness of $f$ is proved. Since $A_{0}$ is evidently metrizable and $\operatorname{dim} A_{0} \leqq 0$ by Theorem 2.1, the implication is completely proved.

To prove the next implication we need the following theorem ${ }^{13)}$.

Theorem 3.1. Let $R$ and $S$ be metric spaces and $f$ a closed continuous mapping of $R$ onto $S$. If $f^{-1}(y)$ consists of exactly $k(<\infty)$ points for every point $y \in S$ and $\operatorname{dim} R \leqq 0$, then we have $\operatorname{dim} S \leqq 0$.

Proof. By Theorem 2.1 we can assume that $R$ is a dense subset of $\lim R_{i}$ obtained from $\left\{R_{i}, f_{j i}: R_{j} \rightarrow R_{i}(j>i)\right\}$ with discrete spaces $R_{i}=\left\{p_{i \alpha} ; \alpha \in A_{i}\right\}$. We can assume that points of $R_{i}$ are linearly-ordered such that for any $p_{i \alpha}, p_{i \beta}$ with $f_{i j}\left(p_{i \alpha}\right) \neq f_{i j}\left(p_{i \beta}\right), i>j$, it holds that $p_{i \alpha}>p_{i \beta}$ if and only if $f_{i j}\left(p_{i \alpha}\right)>f_{i j}\left(p_{i \beta}\right)$. We introduce into points $\left(p_{1 \alpha_{1}}, p_{2 \alpha_{2}}, \cdots\right)$ of $\lim R_{i}$ the lexicographic order with respect to the one of $R_{i}$ just defined. Let $x_{1}(y), \cdots, x_{k}(y) \in R$ be the inverse image of $y \in S$ with $x_{1}(y)<\cdots<x_{k}(y)$ and then $R$ is decomposed into mutually disjoint subsets $T_{i}=\left\{x_{i}(y) ; y \in S\right\}, i=1, \cdots, k$.

We shall show that every $T_{i}$ is an $\mathrm{F}_{\sigma}$. To do so it suffices to prove $T_{1}$ is an $\mathrm{F}_{\sigma}$ since the rest case is proved similarly. Let $r(y), y \in S$, be the smallest

13) This theorem will be generalized in Theorem 3.7. 
integer such that $\pi_{r}\left(x_{1}(y)\right), \cdots, \pi_{r}\left(x_{k}(y)\right)$ are mutually different points of $R_{r}$, where $\pi_{r}: R \rightarrow R_{r}$ is the projection. Let $S_{t}=\{y ; y \in S, r(y) \leqq t\}, t=1,2, \cdots$, and $T_{j t}=$ $T_{j} \cap f^{-1}\left(S_{t}\right)$; then evidently i) $S=\bigcup_{t=1}^{\infty} S_{t}$, ii) $T_{1}=\bigcup_{t=1}^{\infty} T_{1 t}$, iii) $T_{1 t} \subset T_{1, t+1}$. The family $\left\{f\left(V\left(p_{t \alpha}\right)\right) ; V\left(p_{t \alpha}\right)=\left\{x ; x \in R, \pi_{t}(x)=p_{t \alpha}\right\}, \alpha \in A_{t}\right\}$ is a closed covering of $S$ such that the sum of any subfamily is also closed. Let $y$ be an arbitrary point of $S_{t}$ and then it is not hard to see that $W_{y}=S-\bigcup\left\{f\left(V\left(p_{t \alpha}\right)\right) ; \alpha \in A_{t}, y \notin f\left(V\left(p_{t \alpha}\right)\right)\right\}$ is an open set of $S$ which contains $y$ and that $z \in S_{t} \cap W_{y}$ implies $\pi_{t}\left(x_{j}(y)\right)=\pi_{t}\left(x_{j}(z)\right)$ for $j=1, \cdots, k$. Therefore the open set $G_{t y}=\bigcup_{j=2}^{k}\left(f^{-1}(W) \cap V\left(\pi_{t}\left(x_{j}(y)\right)\right)\right)$ is unable to meet $T_{1 t}$. Thus $F_{t}=R-\bigcup\left\{G_{t y} ; y \in S_{t}\right\}$ is a closed set with $F_{t} \supset T_{1 t}$ and $F_{t} \cap\left(\bigcup_{j=2}^{k} T_{j t}\right)=\phi$. Since $H_{j}=\bigcap_{t=j}^{\infty} F_{t}$ is a closed set with $H_{j} \supset T_{1 j}$ and $H_{j} \cap$ $\left(\mathrm{U}_{i=2}^{k} T_{i}\right)=\phi, \quad T_{1}=\bigcup_{j=1}^{\infty} H_{j}$ and $T_{1}$ is an $\mathrm{F}_{\sigma}$. Since $f \mid H_{j}$ is a homeomorphism, $\operatorname{dim} f\left(H_{j}\right) \leqq 0$. Moreover $f\left(H_{j}\right)$ is closed in $S$ and $S=\bigcup_{j=1}^{\infty} f\left(H_{j}\right)$ and hence $\operatorname{dim} S \leqq 0$ by the sum theorem, which completes the proof.

(C) implies (D). Let $A_{0}$ be a metric space with $\operatorname{dim} A_{0} \leqq 0$ and $f$ a closed continuous mapping of $A_{0}$ onto $R$ of order $\leqq n+1$. Let $R_{i}$ be the aggregate of points $x \in R$ such that $f^{-1}(x)$ consists of exactly $i$ points. Then by Theorem 3.1 $\operatorname{dim} R_{i} \leqq 0$. Thus $R$ is the sum of $n+1$ subspaces $R_{i}, i=1, \cdots, n+1$, with $\operatorname{dim} R_{i} \leqq 0$.

(D) implies (E). We prove this by the induction on $n$. When $\operatorname{dim} R \leqq 0$, it is almost evident that Ind $R \leqq 0$.

Let $R$ be the sum of $n+1$ subspaces $R_{i}, i=1, \cdots, n+1$, with $\operatorname{dim} R_{i} \leqq 0$ and assume that Ind $\bigcup_{i=2}^{n+1} R_{i} \leqq n-1$. Let $F$ and $G$ be respectively a closed set and an open set of $R$ with $F \subset G$. Let $H_{1}$ and $H_{2}$ be open sets such that $F \subset H_{1} \subset$ $\bar{H}_{1} \subset H_{2} \subset \bar{H}_{2} \subset G$. Since Ind $R_{1} \leqq 0$, there exists an open and closed subset $U_{1}$ of $R_{1}$ with $R_{1} \cap \bar{H}_{1} \subset U_{1} \subset R_{1} \cap H_{2}$. Let $P_{1}=F \cup U_{1}$ and $P_{2}=(R-G) \cup\left(R_{1}-U_{1}\right)$ and then we get $\left(\bar{P}_{1} \cap P_{2}\right) \cup\left(P_{1} \cap \bar{P}_{2}\right)=\phi$. Hence there exists an open set $U$ of $R$ such that $P_{1} \subset U \subset \bar{U} \subset R-P_{2}$. Then it can easily be seen that $(\bar{U}-U) \cap R_{1}=\phi$ and $F \subset U \subset G$. Since Ind $\bigcup_{i=2}^{n+1} R_{i} \leqq n-1$ and $\bar{U}-U$ is a closed subset of $\bigcup_{i=2}^{n+1} R_{i}$, we get Ind $(\bar{U}-U) \leqq n-1$, which proves Ind $R \leqq n$.

(E) implies (A). This is well-known and has been verified for the first time by N. Vedenisoff [30]. For the proof see for instance [6], [16], [17], [20] or [30].

Theorem 3.2. ${ }^{14)}$ Let $R$ be a metric space. Then the propositions (A), (B), (C), (D), (E) are all equivalent.

TheOREM 3.3. Let $R$ and $S$ be metric spaces with $\operatorname{dim} R \leqq 0$ and $f$ a closed continuous mapping of $R$ onto $S$ of finite order. Then $\operatorname{dim} S+1 \leqq$ the number of integers $i$ with $\left\{y ;\left|f^{-1}(y)\right|=i\right\} \neq \phi$.

This is almost evident by Theorem 3.1 and the implication (D) $\rightarrow(A)$.

Theorem 3.4. Let $R$ be a metric space with $\operatorname{dim} R=n$. Then for any open covering $\mathfrak{U}$ there exists a locally finite closed covering $\mathfrak{F}$ such that

(3.4) $\mathfrak{\vartheta}$ refines $\mathfrak{U}$,

(3.5) order $\mathfrak{\wp} \leqq n+1$,

(3.6) for any $i$ with $1 \leqq i \leqq n+1$ there exists a point $x$ of $R$ with $\operatorname{order}(x, \mathfrak{F})^{15)}=i$.

14) $(\mathrm{A}) \rightleftarrows(\mathrm{D}) \rightleftarrows(\mathrm{E})$ have been proved by Katětov [10] and Morita [16] independently. $(\mathrm{E}) \rightleftarrows(\mathrm{C})$ has been proved by Morita [18]. (B) is a weaker condition which characterizes dimension than that of C.H. Dowker and W. Hurewicz [6].

15) $\operatorname{order}(x, \mathfrak{F})$ is the number of elements of $\mathfrak{F}$ which contain $x$. 
Proof. Let $A_{0}$ be a dense subset of $\lim \left\{A_{i}(i=1,2, \cdots), f_{i j}: A_{i} \rightarrow A_{j}(i>j)\right\}$, where $A_{i}, i=1,2, \cdots$, are topological spaces with discrete topology, and $f$ a closed continuous mapping of $A_{0}$ onto $R$ with order $f \leqq n+1$ such that $\left\{f\left(\pi_{1}^{-1}(\alpha)\right)\right.$; $\left.\alpha \in A_{1}\right\}$ refines $\mathfrak{U}$, where $\pi_{i}, i=1,2, \cdots$, is the projection of $A_{0}$ onto $A_{i}$, and $\left\{f\left(\pi_{i}^{-1}(\alpha)\right) ; \alpha \in A_{i}\right\}$ is locally finite for every $i$. The existence of such $A_{0}$ and $f$ is essentially proved in the proofs of $(\mathrm{A}) \rightarrow(\mathrm{B}) \rightarrow(\mathrm{C})$ of Theorem 3.2. By Theorem 3.3 there exists, for any $i$ with $1 \leqq i \leqq n+1$, a point $y_{i}$ of $R$ with $\operatorname{order}\left(y_{i}, f\right)=i$. Let $j$ be an integer such that the points of $\bigcup\left\{f^{-1}\left(y_{i}\right)\right.$; $i=1, \cdots, n+1\}$ have mutually different representatives in $A_{j}$. Let $\mathfrak{F}=\left\{f\left(\pi_{j}^{-1}(\alpha)\right)\right.$; $\left.\alpha \in A_{j}\right\}$ and then it can easily be seen that $\operatorname{order}\left(y_{i}, \mathfrak{F}\right)=i$ for $i=1, \cdots, n+1$, and that $\mathfrak{F}<\left\{f\left(\pi_{1}^{-1}(\alpha)\right) ; \alpha \in A_{1}\right\}<\mathfrak{H}$. Since $f$ is closed, $\mathfrak{F}$ is a locally finite closed covering of $R$. Thus $\widetilde{\wp}$ satisfies all conditions required, and the theorem is proved.

THEOREM 3.5. ${ }^{16)} \quad$ Let $R$ be a metric space with $\operatorname{dim} R \leqq n$ and $\mathfrak{H}$ be an arbitrary open covering of $R$. Then there exists a sequence of locally finite closed coverings $\mathfrak{H}_{i}=\left\{H_{\alpha} ; \alpha \in A_{i}\right\}, i=1,2, \cdots$, and a sequence of mappings $f_{i+1, i}: A_{i+1} \rightarrow A_{i}$, $i=$ $1,2, \cdots$, which satisfy the following conditions.

(3.7) $\mathfrak{S}_{1}$ refines $\mathfrak{H}$.

(3.8) mesh $\mathfrak{S}_{i} \rightarrow 0$ as $i \rightarrow \infty$.

(3.9) order $\mathfrak{S}_{i} \leqq n+1$ for every $i$.

(3.10) For any $i$ and any $\alpha \in A_{i}, H_{\alpha}=\bigcup\left\{H_{\beta} ; \beta \in f_{i+1, i}^{-1}(\alpha)\right\}$.

(3.11) For any $i$ any $s \leqq n+2, \operatorname{dim} \bigcap_{j=1}^{s}\left\{H_{\alpha(j)} ; \alpha(1), \cdots, \alpha(s)\right.$ are mutually different indices of $\left.A_{i}\right\} \leqq n-s+1$.

Moreover if a sequence of locally finite closed coverings $\mathfrak{S}_{i}, i=1,2, \cdots$, of $R$ satisfies (3.8), (3.9), (3.10), then it satisfies (3.11).

Proof. The existence of a sequence satisfying (3.7), (3.8), (3.9), (3.10) is essentially proved in the proof of the above theorem, if we set $H_{\alpha}=f\left(\pi_{i}^{-1}(\alpha)\right)$ for $\alpha \in A_{i}$.

Let $\mathfrak{S}_{i}, i=1,2, \cdots$, be a sequence of locally finite closed coverings satisfying conditions (3.8), (3.9), (3.10). We show that it satisfies (3.11). Let $\alpha(j), j=$ $1, \cdots, s(\leqq n+2)$, are mutually different indices of $A_{i}$ and put $H=\bigcap_{j=1}^{s} H_{\alpha(j)}$. When $H=\phi, \operatorname{dim} H=-1 \leqq n-s+1$. Let us consider the case $H \neq \phi$. Consider a sequence of locally finite closed coverings $H \cap \mathfrak{S}_{j}, j=i, i+1, \cdots$, of $H$. Let $B_{j}=$ $\left\{\alpha ; \alpha \in A_{j}, H \cap H_{\alpha} \neq \phi, j=i, i+1, \cdots\right.$. Let $g_{j+1, j}=f_{j+1, j} \mid B_{j+1}, j=i, i+1, \cdots$, and then $g_{j+1, j}$ is onto. Let $B_{0}$ be the aggregate of points $a=\left(\alpha_{i}, \alpha_{i+1}, \cdots\right) \in \lim \left\{B_{j}\right.$ $\left.(j=i, i+1, \cdots), g_{j+1, j}\right\}$ such that $\bigcap_{j=i}^{\infty}\left(H \cap H_{\alpha_{j}}\right) \neq \phi$. Let $g$ be a mapping of $B_{0}$ into $H$ defined by $g(a)=\bigcap_{j=1}^{\infty}\left(H \cap H_{\pi_{j}(a)}\right)$, where $\pi_{j}$ is the projection of $B_{0}$ onto $B_{j}$, and then we can know by the same argument as in the proof of $(\mathrm{B}) \rightarrow(\mathrm{C})$ of Theorem 3.2 that $g$ is a closed continuous mapping of $B_{0}$ onto $H$ with order $g \leqq n+1$. Since it can easily be seen by (3.10) that $g\left(\pi_{j}^{-1}(\alpha)\right)=H \cap H_{\alpha}$ for every $\alpha \in B_{j}, j=i, i+1, \cdots$, it holds that $\operatorname{order}(x, g) \geqq s$ for every point $x \in H$ by the inequality $\operatorname{order}\left(x, H \cap \mathfrak{S}_{j}\right) \geqq s$ for $x \in H$ and $j=i, i+1, \cdots$. Since $B_{0}$ has of course $\operatorname{dim} B_{0} \leqq 0$, we get $\operatorname{dim} H \leqq n+1-(s-1)-1=n-s+1$ by Theorem 3.3 and the proof is completed.

Theorem 3.6. Let $R$ be a metric space and $C_{i}, i=1,2, \cdots$, be closed sets of

16) Theorems 3.5 and 3.6 have been proved independently by Prof. Morita. These are also deeply related to Morita $[16 ; 18]$ and Kat ${ }^{\vee}$ tov [11]. 
$R$ with $\operatorname{dim} C_{i}<\infty$ and $\mathfrak{u}$ be an arbitrary open covering of $R$. Then there exist $a$ sequence of locally finite closed coverings $\mathfrak{S}_{i}=\left\{H_{\alpha} ; \alpha \in A_{i}\right\}, i=1,2, \cdots$, and $a$ sequence of mappings $f_{i+1, i}: A_{i+1} \rightarrow A_{i}, i=1,2, \cdots$, which satisfy the following conditions.

(3.12) $\mathfrak{S}_{1}$ refines $\mathfrak{U}$.

(3.13) mesh $\mathfrak{S}_{i} \rightarrow 0$ as $i \rightarrow \infty$.

(3.14) For any $i$ and any $j$, order $\mathfrak{S}_{i} \cap C_{j} \leqq \operatorname{dim} C_{j}+1$.

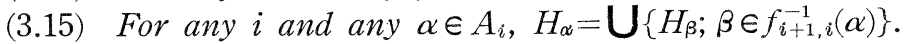

(3.16) For any $i, s\left(\leqq \operatorname{dim} C_{i}+2\right)$ and $t, \operatorname{dim} \bigcap_{j=1}^{s}\left\{H_{\alpha(j)} \cap C_{i} ; \alpha(1), \cdots, \alpha(s)\right.$ are mutually different indices of $\left.A_{t}\right\} \leqq \operatorname{dim} C_{i}-s+1$.

Moreover if a sequence of locally finite closed coverings $\mathfrak{H}_{i}, i=1,2, \cdots$, and $f_{i+1, i}, i=1,2, \cdots$, satisfy the conditions (3.13), (3.14), (3.15), they satisfy (3.16).

Proof. First we construct by the induction a sequence of locally finite closed coverings $\mathfrak{F}_{i}=\left\{F_{\alpha} ; \alpha \in B_{i}\right\}, i=1,2, \cdots$, and a sequence of locally finite open coverings $\mathfrak{H}_{i}=\left\{U_{\alpha} ; \alpha \in B_{i}\right\}, i=1,2, \cdots$, which satisfy the following conditions.

(3.17) $\mathfrak{U}>\mathfrak{F}_{1}>\mathfrak{H}_{1}>\mathfrak{F}_{2}>\mathfrak{H}_{2}>\cdots$.

(3.18) $\operatorname{mesh} \widetilde{\mho}_{i}<1 / i$.

(3.19) For any $i$ and any $j \leqq i$, order $\mathfrak{F}_{i} \cap C_{j} \leqq \operatorname{dim} C_{j}+1$.

Let $\mathfrak{B}_{1}=\left\{V_{\alpha} ; \alpha \in B_{1}\right\}$ be a locally finite open covering of $R$ with mesh $\mathfrak{V}_{1}<1$ such that $\mathfrak{B}_{1}$ refines $\mathfrak{H}$. Let $\mathfrak{W}^{\prime}=\left\{W_{\alpha^{\prime}} ; \alpha \in B_{1}\right\}$ be an open covering of $C_{1}$ such that order $W^{\prime} \leqq \operatorname{dim} C_{1}+1$ and $W_{\alpha^{\prime}} \subset V_{\alpha}$ for every $\alpha \in B_{1}$. Let $W_{\alpha^{\prime \prime}}{ }^{\prime}, \alpha \in B_{1}$, be an open set of $R$ with $W_{\alpha^{\prime \prime}}^{\prime \prime} \cap C_{1}=W_{\alpha^{\prime}}$. Let $W_{\alpha}=\left(W_{\alpha^{\prime \prime}}^{\prime \prime} \cap V_{\alpha}\right) \cup\left(V_{\alpha}-C_{1}\right)$. Then $\mathfrak{W}=$ $\left\{W_{\alpha} ; \alpha \in B_{1}\right\}$ is a locally finite open covering of $R$ and hence there exist a closed covering $\widetilde{F}_{1}=\left\{F_{\alpha} ; \alpha \in B_{1}\right\}$ and an open covering $\mathfrak{H}_{1}=\left\{U_{\alpha} ; \alpha \in B_{1}\right\}$ such that $U_{\alpha} \subset$ $F_{\alpha} \subset W_{\alpha}\left(\subset V_{\alpha}\right)$ for every $\alpha \in B_{1}$. Since $\mathfrak{W} \cap C_{1}=\mathfrak{W}^{\prime}$, order $\mathfrak{F}_{1} \cap C_{1} \leqq \operatorname{order} \mathfrak{W} \cap C_{1}=$ order $\mathfrak{W}^{\prime} \leqq \operatorname{dim} C_{1}+1$.

Now make the induction assumption that there exist locally finite closed coverings $\mathfrak{F}_{1}, \cdots, \mathfrak{F}_{m-1}$ and locally finite open coverings $\mathfrak{H}_{1}, \cdots, \mathfrak{U}_{m-1}$ which satisfy the conditions (3.17), (3.18),(3.19) for $i=1, \cdots, m-1$. Let $\mathfrak{V}_{m}=\left\{V_{\alpha} ; \alpha \in B_{m}\right\}$ be a locally finite open covering of $R$ such that $\mathfrak{B}_{m}$ refines $\mathfrak{H}_{m-1}$ and mesh $\mathfrak{B}_{m}<1 / m$. Let $D_{1}, \cdots, D_{m}$ be a system with $\operatorname{dim} D_{1} \leqq \operatorname{dim} D_{2} \leqq \cdots \leqq \operatorname{dim} D_{m}$ obtained by changing the order of $C_{1}, \cdots, C_{m}$. By the same argument as in the above there exist open coverings $\mathfrak{W}_{i}=\left\{W_{i \alpha} ; \alpha \in B_{m}\right\}, i=1, \cdots, m$, such that $V_{\alpha} \supset W_{1 \alpha} \supset W_{2 \alpha} \supset \cdots \supset W_{m \alpha}$ for every $\alpha \in B_{m}$ and $\operatorname{order} \mathfrak{W}_{i} \cap D_{i} \leqq \operatorname{dim} D_{i}+1$ for $i=1, \cdots, m$. Since order $\mathfrak{W}_{i} \cap D_{j} \leqq$ order $\mathfrak{W}_{j} \cap D_{j}$ for $j \leqq i$, we get order $\mathfrak{W}_{m} \cap C_{i} \leqq$ order $\mathfrak{W}_{i_{1}} \cap C_{i} \leqq \operatorname{dim} C_{i}+1$ for $1, \cdots, m$, where $C_{i}=D_{i_{1}}$. Since $\mathfrak{W}_{m}=\left\{W_{m \alpha} ; \alpha \in B_{m}\right\}$ is locally finite, there exist a closed covering $\widetilde{F}_{m}=\left\{F_{\alpha} ; \alpha \in B_{m}\right\}$ and an open covering $\mathfrak{H}_{m}=\left\{U_{\alpha} ; \alpha \in B_{m}\right\}$ such that $U_{\alpha} \subset F_{\alpha} \subset W_{\alpha}\left(\subset V_{\alpha}\right)$ for every $\alpha \in B_{m}$. It is obvious that i) order $\widetilde{\mho}_{m} \cap C_{i} \leqq \operatorname{dim} C_{i}+1$ for $i \leqq m$, ii) $\mathfrak{U}_{m-1}>\mathfrak{F}_{m}>\mathfrak{U}_{m}$, iii) mesh $\mathfrak{\mho}_{m}<$ $1 / m$, which completes the induction.

Now define $f_{i+1, i}: B_{i+1} \rightarrow B_{i}$ in such a way that $f_{i+1, i}(\alpha)=\beta$ yields $F_{\alpha} \subset F_{\beta}$. Let $B_{0}$ be the aggregate of points $a=\left(\alpha_{1}, \alpha_{2}, \cdots\right)$ of $\lim \left\{B_{i}(i=1,2, \cdots), f_{i+1, i}\right\}$ such that $\bigcap_{i=1}^{\infty} F_{\alpha_{i}} \neq \phi$. Let $f$ be a mapping of $B_{0}$ into $R$ defined by $f(a)=$ $\bigcap_{i=1}^{\infty} F_{\pi_{i}(u)}$, where $\pi_{i}$ is the projection of $B_{0}$ into $B_{i}$. Then we can know by the same argument as in the proof of $(\mathrm{B}) \rightarrow(\mathrm{C})$ of Theorem 3.2 that $f$ is a closed continuous mapping of $B_{0}$ onto $R$. 
Let

(3.20) $\left.\mathfrak{S}_{i}=\left\{H_{\alpha}=f\left(\pi_{i}^{-1} \alpha\right)\right) ; \alpha \in A_{i}=\pi_{i}\left(B_{0}\right)\right\}, i=1,2, \cdots$,

and then they form a sequence of locally finite closed coverings which satisfies (3.12), (3.13), (3.15). Since $\mathfrak{S}_{i}$ refines $\mathfrak{F}_{i}$ for every $i$, we get i) order $\mathfrak{S}_{i} \cap C_{j} \leqq$ order $\widetilde{\mho}_{i} \cap C_{j} \leqq \operatorname{dim} C_{j}+1$ when $i \geqq j$, ii) order $\mathfrak{S}_{i} \cap C_{j} \leqq$ order $\mathfrak{S}_{j} \cap C_{j} \leqq \operatorname{dim} C_{j}+1$ when $i<j$ by (3.15) and (3.19). Thus the sequence (3.20) satisfies (3.14).

When a sequence of locally finite closed coverings satisfies (3.13), (3.14), (3.15), we can know that it satisfies also (3.16) by the same argument as in the proof of the preceding theorem. Thus the theorem is completely proved.

Theorem 3.7. Let $R$ and $S$ be metric spaces and $f$ a closed continuous mapping of $R$ onto $S$. If $f^{-1}(y)$ consists of exactly $k(<\infty)$ points for every point $y \in S$, then we have $\operatorname{dim} R=\operatorname{dim} S$.

Proof. Since it holds in general that $\operatorname{dim} R \leqq \operatorname{dim} S+\sup \left\{\operatorname{dim} f^{-1}(y) ; y \in S\right\}$ by Morita [17, Theorem 2] or Nagami [20, Theorem 2], we get $\operatorname{dim} R \leqq \operatorname{dim} S$.

Conversely let us prove that $\operatorname{dim} S \leqq \operatorname{dim} R$. Let $\mathfrak{F}_{i}=\left\{F_{\alpha} ; \alpha \in A_{i}\right\}, \mathrm{i}=1,2, \cdots$, be locally finite closed coverings of $R$ such that mesh $\widetilde{\mho}_{i} \rightarrow 0$ as $i \rightarrow \infty$. Then $f\left(\mathfrak{\mho}_{i}\right)=\left\{f\left(F_{\alpha}\right) ; \alpha \in A_{i}\right\}$ is a point-finite closed covering of $S$. Let $y$ be an arbitrary point of $S$ and $B_{i}$ the subset of $A_{i}$ such that $\alpha \in B_{i}$ if and only if $y \notin f\left(F_{\alpha}\right)$. Since $\bigcup\left\{F_{\alpha} ; \alpha \in B_{i}\right\}$ is closed, $V=S-f\left(\bigcup\left\{F_{\alpha} ; \alpha \in B_{i}\right\}\right)=S-\bigcup\left\{f\left(F_{\alpha}\right) ; \alpha \in B_{i}\right\}$ is an open neighborhood of $y$. If $f\left(F_{\alpha}\right) \cap V \neq \phi$, then $\alpha \notin B_{i}$. Since $A_{i}-B_{i}$ is finite, $V$ intersects only a finite number of $f\left(F_{a}\right), \alpha \in A_{i}$. Thus $f\left(\mathfrak{F}_{i}\right)$ is locally finite.

Let $y$ be an arbitrary point of $S$ and $\left\{x_{1}, \cdots, x_{k}\right\}$ the inverse image of $y$. Then there exists $i$ such that when $x_{s} \in F_{\alpha} \in \mathfrak{F}_{i}, x_{i} \in F_{\beta} \in \mathfrak{F}_{i}, s \neq t$, then $F_{\alpha} \cap F_{\beta}=$ $\phi$. Select $F_{\alpha(j)} \in \mathfrak{F}_{i}, j=1, \cdots, k$, such that $x_{j} \in F_{\alpha(j)}$ and put $F(y)=\bigcap_{j=1}^{k} f\left(F_{\alpha(j)}\right)$; then $F(y)$ is a closed set of $S$ which contains $y$. Since $F_{\alpha_{(1)}} \cap f^{-\mathrm{T}}(F(y))$ is a closed set of $R$ and $f \mid F_{\alpha(1)} \cap f^{-1}(F(y))$ is a homeomorphism of $F_{\alpha(1)} \cap f^{-1}(F(y))$ onto $F(y), \operatorname{dim} F(y)=\operatorname{dim} F_{\alpha^{(1)}} \cap f^{-1}(F(y)) \leqq \operatorname{dim} R$. Since $\{F(y) ; y \in S\}$ is a closed covering of $S$ which is a countable sum of locally finite subfamilies, $\operatorname{dim} S=$ $\sup \{\operatorname{dim} F(y) ; y \in S\} \leqq \operatorname{dim} R$ by Morita [16, Theorem 5.2] or by Nagami [19, Theorem 2], which completes the proof.

A slight modification of the above theorem is the following.

THeOREM 3.8. Let $R$ and $S$ be metric spaces and $f$ a closed continuous mapping of $R$ onto $S$. If the boundary of $f^{-1}(y)$ consists of exactly $k(<\infty)$ points for every point $y \in S$, then we have $\operatorname{dim} S \leqq \operatorname{dim} R$.

Proof. When $R=\phi$, then $S=\phi$ and evidently $\operatorname{dim} S=\operatorname{dim} R$. Let up prove the theorem for the case when $R \neq \phi$. When $k=0$, select a point $x(y) \in f^{-1}(y)$ for every $y \in S$. Then it can easily be seen that $\{x(y) ; y \in S\}$ is a closed set of $R$ and hence it is homeomorphic to $S$ under $f \mid\{x(y) ; y \in S\}$. Therefore $\operatorname{dim} S=$ $\operatorname{dim}\{x(y) ; y \in S\} \leqq \operatorname{dim} R$.

When $k>0, R_{1}=R-\bigcup$ the open kernel of $\left.f^{-1}(y) ; y \in S\right\}$ is a closed set of $R$ and $f_{1}=f \mid R_{1}$ is a closed continuous mapping of $R_{1}$ onto $S$ such that $f_{1}^{-1}(y)$ consists of exactly $k$ points for every point $y \in S$. Hence Theorem 3.7 can be applied and we get $\operatorname{dim} S=\operatorname{dim} R_{1} \leqq \operatorname{dim} R$, which completes the proof.

When a metric space $R$ is the sum of countable subspaces $R_{i}$ with $\operatorname{dim} R_{i} \leqq 0$, we call $\operatorname{dim} R \leqq \boldsymbol{\aleph}_{0}$. Under this definition we get the following theorem. 
Theorem 3.9. Let $R$ and $S$ be metric spaces and $f$ a closed continuous mapping of $R$ onto $S$. If the boundary of $f^{-1}(y)$ is finite for every point $y \in S$ and $\operatorname{dim} R \leqq \boldsymbol{\aleph}_{0}$, then we have $\operatorname{dim} S \leqq \boldsymbol{\aleph}_{0}$.

Proof. Let $S_{i}, i=0,1,2, \cdots$, be the aggregate of points $y \in S$ such that the boundary of $f^{-1}(y)$ consists of exactly $i$ points. Then $S=\bigcup_{i=0}^{\infty} S_{i}$ and $\operatorname{dim} S_{i} \leqq$ $\operatorname{dim} R \leqq \boldsymbol{\aleph}_{0}$ by Theorem 3.8 and hence $\operatorname{dim} S \leqq \boldsymbol{\aleph}_{0}$.

\section{$\S 4$. Open mappings whose order are point-wise finite}

Theorem 4.1. Let $R$ and $S$ be paracompact Hausdorff spaces and $f$ an open continuous mapping of $R$ onto $S$. If $f^{-1}(y)$ is finite for every point $y \in S$, then we have $\operatorname{dim} R=\operatorname{dim} S$. If moreover the paracompactness of $R$ and $S$ is hereditary, we have Ind $R=\operatorname{Ind} S$.

Proof. Let $S_{i}$ be the aggregate of points $y \in S$ such that $f^{-1}(y)$ consists of exactly $i$ points; then $S=\bigcup_{i=1}^{\infty} S_{i}$. Let $\left\{x_{1}, \cdots, x_{i}\right\}$ be the inverse image of $y$, $y \in S_{i}$, under $f$. Let $V_{j}$ be an open neighborhood of $x_{j}, j=1, \cdots, i$, such that $V_{j} \cap V_{k}=\phi$ if $j \neq k$. Since $f$ is open, $V=\bigcap_{j=1}^{i} f\left(V_{j}\right)$ is an open neighborhood of $y$. Since for every point $y \in V f^{-1}(y)$ consists of at least $i$ points, $V \subset \bigcup_{k=i}^{\infty} S_{k}$. Thus

(4.1) $\bigcup_{k=1}^{i} S_{k}$ is closed in $S$ for every $i$.

Let $R_{i}=f^{-1}\left(S_{i}\right)$ and consider $f_{i}=f \mid R_{i}$ and then $f_{i}$ is an open continuous mapping of $R_{i}$ onto $S_{i}$ such that $f_{i}^{-1}(y)$ consists of exactly $i$ points for every point $y \in S_{i}$. Let $\left\{x_{1}, \cdots, x_{i}\right\}$ be the inverse image of $y \in S_{i}$ under $f_{i}$. Let $W_{j}$ be an open neighborhood of $x_{j}, j=1, \cdots, i$, such that $W_{j} \cap W_{k}=\phi$ whenever $j \neq k$. Since $f_{i}$ is open, $W=\bigcap_{j=1}^{i} f_{i}\left(W_{j}\right)$ is an open neighborhood of $y$. Since $W_{j} \cap f^{-1}(W)$ is homeomorphic to $W$ under $f_{i}, j=1, \cdots, i$, and the covering dimension of paracompact Hausdorff spaces is completely determined by the local covering dimension by C. H. Dowker [5] or Nagami [19, Theorem 1], we get $\operatorname{dim} R_{i}=$ $\operatorname{dim} S_{i}$. Since $\operatorname{dim} R=\sup \operatorname{dim} R_{i}$ and $\operatorname{dim} S=\sup \operatorname{dim} S_{i}$ by (4.1) (cf. for instance [20, Lemma 3]), we get $\operatorname{dim} R=\operatorname{dim} S$.

When $R$ and $S$ are hereditarily paracompact Hausdroff spaces, we get Ind $R=$ Ind $S$ since i) the large inductive dimension of such spaces is completely determined by the local large inductive dimension by C. H. Dowker [5], ii) Ind $R=$ sup Ind $R_{i}$ and Ind $S=\sup$ Ind $S_{i}$ by C. H. Dowker [4]. Thus the theorem is completely proved.

\section{$\S 5$. Cuts}

Definition 5.1. Let $R$ and $S$ be topological spaces. Let $\mathfrak{F}=\left\{F_{\alpha} ; \alpha \in A\right\}$ and $\mathfrak{S}=\left\{H_{\alpha} ; \alpha \in A\right\}$ be respectively locally finite closed coverings of $R$ and $S$. Let $f$ be a mapping of $R$ onto $S$. Let $r$ be a positive integer. If the following conditions are satisfied, $(R, \mathfrak{F}, f)$ is called the cut of order $r$ of $(S, \mathfrak{g})$.

(5.1) For every $\alpha \in A, f \mid F_{\alpha}$ is a homeomorphism of $F_{\alpha}$ onto $H_{\alpha}$.

(5.2) If order $(y, \mathfrak{g}), y \in S$, is greater than $r, f^{-1}(y)$ consists of one and only one point.

(5.3) If $r_{1}=\operatorname{order}(y, \mathfrak{S}), y \in S$, is not greater than $r, f^{-1}(y)$ consists of exactly $r_{1}$ points. 
$R$ is called the cut-space of order $r$ obtained from $(S, \mathfrak{Z}), \Im$ the derived covering of order $r$ and $f$ the cut-mapping.

It is to be noted that $f$ is a closed continuous mapping by the condition (5.1) and the local finiteness of $\widetilde{F}$ and $\mathfrak{S}$.

Theorem 5.2. Let $S$ be an arbitrary topological space, $\mathfrak{g}=\left\{H_{\alpha} ; \alpha \in A\right\}$ an arbitrary locally finite closed covering of $S$ and $r$ an arbitrary positive integer. Then there exists a cut $\left(R, \mathfrak{F}=\left\{F_{\alpha} ; \alpha \in A\right\}, f\right)$ of order $r$ of $(S, \mathfrak{S})$. Moreover the cut is essentially unique: If $\left(T, \mathcal{R}=\left\{K_{\alpha} ; \alpha \in A\right\}, \mathrm{g}\right)$ is another cut of order $r$ of $(S, \mathfrak{S})$, there exists a homeomorphism $h$ of $R$ onto $T$ such that $h\left(F_{\infty}\right)=K_{\infty}$ and $g h\left|F_{\alpha}=f\right| F_{\alpha}$ for every $\alpha \in A$.

Proof. First let us construct a cut $(R, \mathfrak{F}, f)$ of order $r$ of $(S, \mathfrak{S})$. Let $R_{0}$ be the mutually disjoint union of $C_{\alpha}, \alpha \in A$, where every $C_{\alpha}$ is a copy of $H_{\alpha}$. Let $f_{0 \alpha}: C_{\alpha} \rightarrow H_{\alpha}, \alpha \in A$, be a copy-mapping. ${ }^{17)}$ Let $f_{0}$ be a mapping of $R_{0}$ onto $S$ such that for every $\alpha \in A f_{0} \mid C_{\alpha}=f_{0 \alpha}$. Define the topology of $R_{0}$ as follows: A subset $B$ of $R_{0}$ is closed if and only if for every $\alpha \in A f_{0}\left(B \cap C_{\alpha}\right)$ is closed in $H_{a}$ (and hence closed in $S$ ). It can easily be seen that $R_{0}$ with this topology is a topological space. Then $f_{0}$ is a closed continuous mapping such that for every $\alpha \in A f_{0} \mid C_{\alpha}$ is a homeomorphism of $C_{\alpha}$ onto $H_{\alpha}$. Let $H=\{x ; x \in S$, $\operatorname{order}(x, \mathfrak{S})>r\}$; then $H$ is a closed subset of $S$ by the local finiteness of $\mathfrak{S}$. Let $R_{1}$ be a copy of $H$ and $f_{1}: R_{1} \rightarrow H$ a copy-mapping. Let $R_{2}$ be a copy of $R_{0}-f_{0}{ }^{-1}(H)$ and $f_{2}: R_{2} \rightarrow R_{0}-f_{0}^{-1}(H)$ a copy-mapping. Let $R$ be the disjoint union of $R_{1}$ and $R_{2}$. Let $f_{3}$ be a mapping of $R_{0}$ onto $R$ such that i) when $x \in f_{0}^{-1}(H), f_{3}(x)=f_{1}^{-1}\left(f_{0}(x)\right)$, ii) when $x \in R_{0}-f_{0}^{-1}(H), f_{3}(x)=f_{2}^{-1}(x)$. Let $f$ be a mapping of $R$ onto $S$ such that i) when $x \in R_{1}, f(x)=f_{1}(x)$, ii) when $x \in R_{2}, f(x)=$ $f_{0}\left(f_{2}(x)\right)$. Let $F_{\alpha}=f_{3}\left(C_{\alpha}\right), \alpha \in A$; then $\widetilde{\mho}=\left\{F_{\alpha} ; \alpha \in A\right\}$ is a covering of $R$ such that $f\left(F_{\alpha}\right)=H_{\alpha}$ for every $\alpha \in A$.

Define the topology of $R^{18)}$ as follows: A subset $B$ of $R$ is closed if and only if for every $\alpha \in A f\left(B \cap F_{\alpha}\right)$ is closed in $H_{a}$ (and hence closed in $S$ ). Then it can easily be seen that $R$ is a topological space.

Let $B$ be a closed subset of $R$. Since $f(B)=f\left(\mathbf{U}_{\alpha}\left(B \cap F_{\alpha}\right)\right)=\mathbf{U}_{\alpha} f\left(B \cap F_{\alpha}\right), f(B)$ is closed in $S$, which shows the closedness of $f$. Let $C$ be a closed set of $S$; then $f^{-1}(C)$ is closed in $R$ by the equality $f\left(f^{-1}(C) \cap F_{\alpha}\right)=C \cap H_{\alpha}$, which shows the continuity of $f$. Thus $f$ is a closed continuous mapping of $R$ onto $S$.

Let $F_{\alpha}$ and $F_{\beta}$ be arbitrary elements of $\widetilde{\jmath}$; then $f\left(F_{a} \cap F_{\beta}\right)$ is $H_{\alpha}$ or $H_{\alpha} \cap$

17) When there is a one-to-one mapping $f_{0 \alpha}: C_{\alpha} \rightarrow H_{\alpha}, C_{\alpha}$ is called a copy of $H_{\infty}$ and $f_{0 \alpha}$ a copy-mapping for the brevity.

18) It is to be noted that the quotient topology $\mathrm{Q}$ of $R$ obtained from $f_{3}: R_{0} \rightarrow R$ is the same as the topology $\mathrm{P}$ defined in the proof: i) Let $F$ be a closed subset of $R$ with the topology Q. Then by the definition of $\mathrm{Q} f_{3}^{-1}(F)$ is closed and hence $f_{3}^{-1}(F) \cap C_{\alpha}$ is closed for any $\alpha \in A$. Since $f_{0}\left(f_{3}^{-1}(F) \cap C_{\alpha}\right)=f\left(f_{3}\left(f_{3}^{-1}(F) \cap C_{x}\right)\right)=f\left(F \cap f_{3}\left(C_{\alpha}\right)\right)=f\left(F \cap F_{\alpha}\right), f\left(F \cap F_{\alpha}\right)$ is closed by the closedness of $f_{0}$. Thus $F$ is closed in $R$ with the topology P. ii) Conversely let $F$ be a closed subset of $R$ with the topology $\mathrm{P}$; then $f\left(F \cap F_{\alpha}\right)=f_{0}\left(f_{3}{ }^{-1}(F) \cap C_{\boldsymbol{x}}\right)$ is closed for any $\alpha \in A$. Sincd $f_{0} \mid C_{\alpha}: C_{\alpha} \rightarrow H_{\alpha}$ is a homeomorphism, $f_{3}^{-1}(F) \cap C_{\alpha}$ is closed in $C_{\alpha}$. Hence $f_{3}^{-1}(F) \cap C_{\alpha}$ is closed in $R_{0}$. By the local finiteness of $\left\{C_{\alpha} ; \alpha \in A\right\}, f_{3}^{-1}(F)$ is closed in $R_{0}$, which shows the closedness of $F$ in the topology $\mathrm{Q}$.

It is also to be noted that the topology of $S$ is the same as the quotient topology obtained from $f_{0}: R_{0} \rightarrow S$. This fact is a trivial consequence of the closedness and the continuity of $f_{0}: R_{0} \rightarrow S$ with the preasigned topology. 
$H_{\beta} \cap H$ according to $\alpha=\beta$ or $\alpha \neq \beta$. In both cases $f\left(F_{\alpha} \cap F_{\beta}\right)$ is closed, which shows the closedness of $F_{\alpha}$.

Let $x$ be an arbitrary point of $R$ and $V$ a neighborhood of $f(x)$ which meets only a finite number of elements of $\mathfrak{S}=\left\{H_{\alpha} ; \alpha \in A\right\}$. It can easily be seen that $V \cap H_{\alpha} \neq \phi$ if and only if $f^{-1}(V) \cap F_{\alpha} \neq \phi$. Hence $f^{-1}(V)$ meets only a finite number of elements of $\widetilde{F}=\left\{F_{\alpha} ; \alpha \in A\right\}$. Since $f$ is continuous, $f^{-1}(V)$ is a neighborhood of $x$. Thus the local finiteness of $\mathscr{F}$ is proved.

Since it is evident that $(R, \mathfrak{F}, f)$ satisfies $(5.1),(5.2),(5.3),(R, \mathfrak{F}, f)$ is the desired cut of $(S, \mathfrak{S})$.

Let $\left(T, \mathscr{R}=\left\{K_{\alpha} ; \alpha \in A\right\}\right.$, g) be another cut of order $r$ of $(S, \mathfrak{s})$. Let $f_{\alpha}=f \mid F_{\alpha}$ and $g_{\alpha}=g \mid K_{\alpha}, \alpha \in A$; then $h_{\alpha}=g_{\alpha}{ }^{-1} f_{\alpha}$ is a homeomorphism of $F_{\alpha}$ onto $K_{\alpha}$. Let $\alpha$ and $\beta$ be different indices of $A$ with $F_{\alpha} \cap F_{\beta} \neq \phi$. Then $F_{\alpha} \cap F_{\beta} \subset R_{1}$. Let $T_{1}=g^{-1}(H)$ and $g_{1}=g \mid T_{1}$; then $g_{1}$ is a homeomorphism of $T_{1}$ onto $H$. Since $h_{\alpha}\left|F_{\alpha} \cap F_{\beta}=g_{1}^{-1} f_{1}\right| F_{\alpha} \cap F_{\beta}$ and $h_{\beta}\left|F_{\alpha} \cap F_{\beta}=g_{1}^{-1} f_{1}\right| F_{\infty} \cap F_{\beta}$, we can define a mapping $h$ such that $h \mid F_{\alpha}=h_{\alpha}$ for every $\alpha \in A$. Since $\mathfrak{F}$ is locally finite and $h$ is one-to-one onto and $h \mid F_{\alpha}=h_{\alpha}$ is a homeomorphism, $h$ is a homeomorphism of $R$ onto $T$. Evidently $h\left(F_{\alpha}\right)=K_{\alpha}$ and $g h\left|F_{\alpha}=f\right| F_{\alpha}$ for every $\alpha \in A$ and the theorem is completely proved.

REMARK 5.3. It is to be noted that when $S$ is a metric space, $R$ is also metrizable by J. Nagata's metrization theorem [25, Theorem 2]: A topological space is metrizable if it is covered by a locally finite closed covering each of whose elements is metrizable.

REMARK 5.4. Let $y$ be arbitrary point of $S$; then $f^{-1}(y) \cap F_{\alpha}$ is a single point or the empty set for every $\alpha \in A$ by the condition (5.1). Hence if $r_{1}=$ $\operatorname{order}(y, \mathfrak{H}) \leqq r$, then $\operatorname{order}(x, \widetilde{F})=1$ for any point $x \in f^{-1}(y)$ by the condition (5.3).

The following lemma will be used in the succeeding paragraph.

Lemma 5.5. Let $R$ and $S$ be topological spaces, $\mathfrak{F}=\left\{F_{\alpha} ; \alpha \in A\right\}$ and $\mathfrak{g}=$ $\left\{H_{\alpha} ; \alpha \in A\right\}$ respectively locally finite closed coverings of $R$ and $S$, and $\varphi$ a closed continuous mapping of $R$ onto $S$ such that $\varphi\left(F_{\alpha}\right)=H_{\alpha}$ for every $\alpha \in A$. Let $\left(R_{1}, \widetilde{\mho}_{1}=\left\{F_{1 \alpha} ; \alpha \in A\right\}, f\right)$ and $\left(S_{1}, \mathfrak{S}_{1}=\left\{H_{1 \alpha} ; \alpha \in A\right\}, g\right)$ be respectively the cuts of order $r$ of $(R, \mathfrak{F})$ and $(S, \mathfrak{H})$. Then there is one and only one closed continuous mapping $\psi$ of $R_{1}$ onto $S_{1}$ such that $\varphi f=g \psi$.

Proof: First let us prove the existence of $\psi$. Let, for every $\alpha \in A, \varphi_{a}=$ $\varphi\left|F_{\alpha}, f_{\alpha}=f\right| F_{1 \alpha}$ and $g_{\alpha}=g \mid H_{1 \alpha}$. Let $\alpha$ and $\beta$ be different indices of $A$ with $F_{1 \alpha} \cap F_{1 \beta} \neq \phi$ and $x$ an arbitrary point of $F_{1 \alpha} \cap F_{1 \beta}$. Then $\operatorname{order}(f(x), \widetilde{F})>r$ and hence order $\left(\varphi(f(x))\right.$, S) $>r$. Therefore $g^{-1}(\varphi(f(x)))$ is a single point and there exists $y \in S_{1}$ such that $y=g^{-1}(\varphi(f(x)))$. Let $f_{0}=f\left|F_{1 \alpha} \cap F_{1 \beta}, \varphi_{0}=\varphi\right| f\left(F_{1 \alpha} \cap F_{1 \beta}\right)$ and $h=g_{\alpha}{ }^{-1} \mid \varphi\left(f\left(F_{1 \alpha} \cap F_{1 \beta}\right)\right)$. Then $g_{\alpha^{-1}} \varphi_{\alpha} f_{\alpha}\left|F_{1 \alpha} \cap F_{1 \beta}=h \varphi_{0} f_{0}=g_{\beta}{ }^{-1} \varphi_{\beta} f_{\beta}\right| F_{1 \alpha} \cap F_{1 \beta}$. Thus we can define $\psi: R_{1} \rightarrow S_{1}$ in such a way that $\psi \mid F_{1 \alpha}=g_{\alpha}^{-1} \varphi_{\alpha} f_{\alpha}$ for every $\alpha \in A$. Let $\psi_{\alpha}=\psi \mid F_{1 \alpha}, \alpha \in A$; then $\psi_{\alpha}=g_{\alpha^{-1}} \varphi_{\alpha} f_{\alpha}$. Since $g_{\alpha}$ is a homeomorphism, $g_{\alpha} \psi_{\alpha}=$ $\varphi_{\alpha} f_{\alpha}$ for every $\alpha \in A$. In other words $g \psi\left|F_{1 \alpha}=\varphi f\right| F_{1 \alpha}$ for every $\alpha \in A$. Hence $g \psi=\varphi f$. Since $\psi_{\alpha}$ is closed continuous for every $\alpha \in A, \psi$ is closed continuous by the local finiteness of $\widetilde{\mho}_{1}$ and $\mathfrak{H}_{1}$.

Let $\psi_{1}$ be another closed continuous mapping of $R_{1}$ onto $S_{1}$ such that $g \phi_{1}=$ $\varphi f$. Then for every $\alpha \in A g \psi_{1}\left|F_{1 \alpha}=\varphi f\right| F_{1 \alpha}$ and hence $\psi_{1} \mid F_{1 \alpha}=g_{\alpha^{1}}{ }^{-1} \varphi_{\alpha} f_{\alpha}$, which proves $\psi_{1}=\psi$. 


\section{§. W. Hurewicz's problem}

In $\S 3$ we have proved that a metric space $R$ has dimension $\leqq n$ if and only if there exist a metric space $A_{0}$ with $\operatorname{dim} A_{0} \leqq 0$ and a closed continuous mapping $f$ of $A_{0}$ onto $R$ with order $f \leqq n+1$. On the other hand we know that if there exist a metric space $S$ and a closed continuous mapping $g$ of $S$ onto a metric $R$ with order $g \leqq m+1$, then $\operatorname{dim} R \leqq \operatorname{dim} S+m$ (cf. Morita [17]). In consideration of these facts the following question is naturally raised: Let $R$ be a metric space with $\operatorname{dim} R=n$. Are there for every $m, 0<m<n$, a metric space $T$ with $\operatorname{dim} T=m$ and a closed continuous mapping $\pi_{0}$ of $T$ onto $R$ with order $\pi_{0} \leqq n-m+1$ ? This question was raised by W. Hurewicz [9] and solved for the case of separable metric spaces by J.H. Roberts [26]. We shall now construct $T$ and $\pi_{0}$ with the above properties for general metric space $R$ with the aid of the concept of the cuts defined in the preceding paragraph. A figure and diagrams will help us to clarify our construction.

i) Let $R=R_{0}$ be a metric space with $\operatorname{dim} R_{0}=n$. Let $m$ be an arbitrary integer with $0 \leqq m<n$. By Theorem 3.5 there exist a sequence of locally finite closed coverings $\mathfrak{F}_{0 i}=\left\{F\left(0, \alpha_{i}\right) ; \alpha_{i} \in A_{i}\right\},{ }^{19)} i=1,2, \cdots$, and a sequence of mappings $f_{i+1, i}: A_{i+1} \rightarrow A_{i}, i=1,2, \cdots$, which satisfy the following conditions.

(6.1) mesh $\widetilde{\mho}_{0 i} \rightarrow 0$ as $i \rightarrow \infty$.

(6.2) order $\widetilde{F}_{0 i} \leqq n+1$ for every $i$.

(6.3) For any $i$ and any $\alpha_{i} \in A_{i}, F\left(0, \alpha_{i}\right)=\bigcup\left\{F\left(0, \alpha_{i+1}\right) ; \alpha_{i+1} \in f_{i+1, i}^{-1}\left(\alpha_{i}\right)\right\}$.

(6.4) For any $i$ and any $s \leqq n+2, \operatorname{dim} \bigcap_{j=1}^{s}\{F(0, \alpha(j)) ; \alpha(1), \cdots, \alpha(s)$ are mutually different indices of $\left.A_{i}\right\} \leqq n-s+1$.

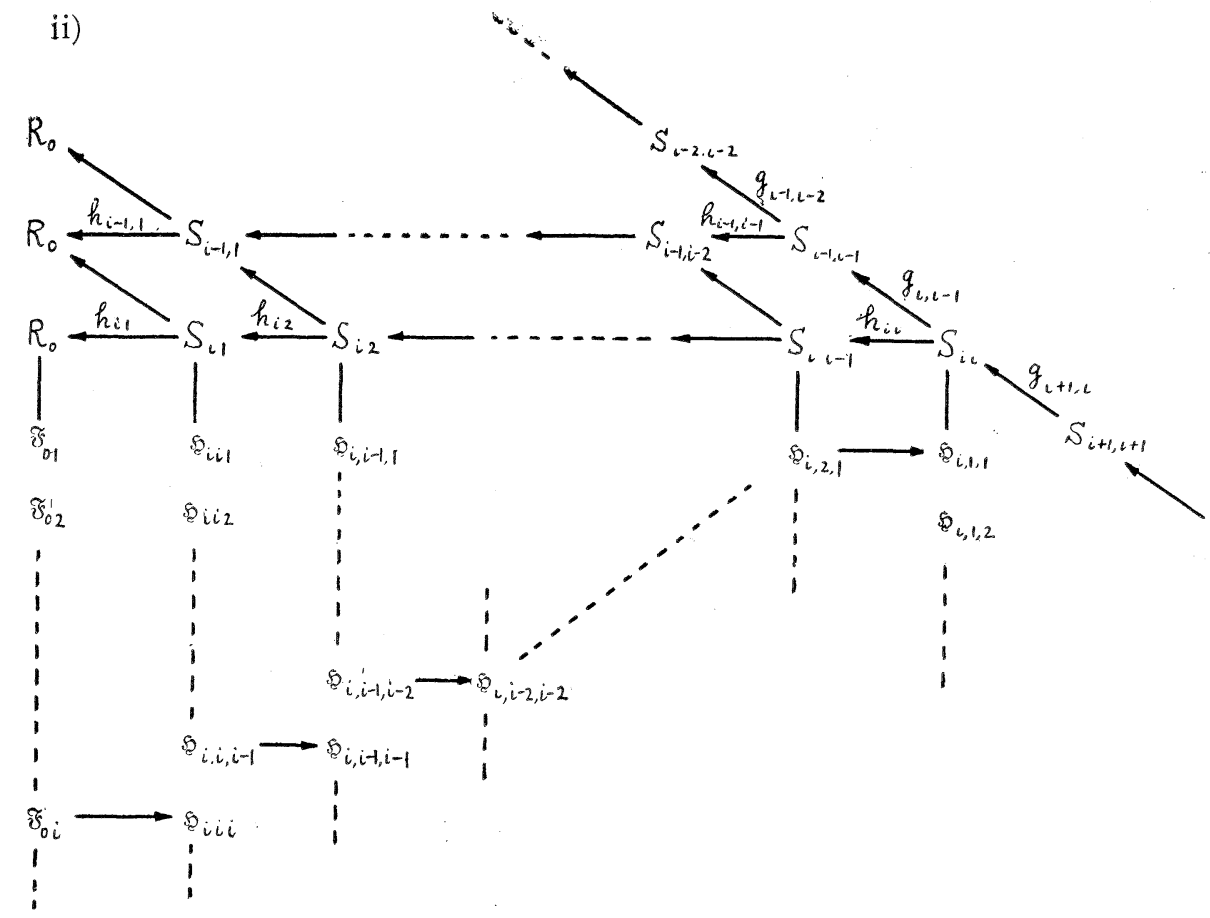

Diagram 1

19) In the following $\alpha_{i}$ always indicates an element of $A_{i}$. 
Let $\left(S_{i 1}, \mathfrak{S}_{i i i}=\left\{H\left(i, i, \alpha_{i}\right) ; \alpha_{i} \in A_{i}\right\}, h_{i 1}\right)$ is the cut of order $r=n-m+1$ of $\left(R_{0}, \mathfrak{F}_{0 i}\right)$. Let

(6.5) $H\left(i, i, \alpha_{j}\right)=\mathbf{U}\left\{H\left(i, i, \alpha_{i}\right) ; f_{i j}\left(\alpha_{i}\right)=\alpha_{j}\right\}$ if $j<i$,

and

(6.6) $H\left(i, i, \alpha_{j}\right)=h_{i 1}^{-1}\left(F\left(0, \alpha_{j}\right)\right) \cap H\left(i, i, f_{j i}\left(\alpha_{j}\right)\right)$ if $j>i$, where $f_{j i}, j>i$, is $f_{i+1, i} \cdots f_{j-1, j-2} f_{j, j-1}$. Then $\mathfrak{g}_{i i j}=\left\{H\left(i, i, \alpha_{j}\right) ; \alpha_{j} \in A_{j}\right\}, j=1,2, \cdots$, is a sequence of locally finite closed coverings of $S_{i 1}$ which satisfies the following conditions.

(6.7) For any point $p$ of $S_{i 1}$ and any neighborhood $U$ of $p$, there exists $\mathfrak{H}_{i i j}$ such that $\operatorname{Star}\left(p, \mathfrak{S}_{i i j}\right) \subset U$.

(6.8) For any $j$ and any $\alpha_{j} \in A_{j}, H\left(i, i, \alpha_{j}\right)=\bigcup\left\{H\left(i, i, \alpha_{j+1}\right) ; f_{j+1, j}\left(\alpha_{j+1}\right)=\alpha_{j}\right\}$.

(6.9) For any $j \geqq i$ and any $\alpha_{j} \in A_{j}, h_{i 1} \mid H\left(i, i, \alpha_{j}\right)$ is a homeomorphism of $H\left(i, i, \alpha_{j}\right)$ onto $F\left(0, \alpha_{j}\right)$.

iii) We construct by a successive process the cuts $\left(S_{i j}, \mathfrak{S}_{i, i-j+1, i-j+1}, h_{i j}\right)$, $j=2, \cdots, i$, and sequences of locally finite closed coverings $\mathfrak{S}_{i, i-j+1, k}=$ $\left\{H\left(i, i-j+1, \alpha_{k}\right) ; \alpha_{k} \in A_{k}\right\}, \quad k=1,2, \cdots$, of $S_{i j}, \quad j=2, \cdots, i$, which satisfy the following conditions.

(6.10) $\left(S_{i j}, \mathfrak{H}_{i, i-j+1, i-j+1}, h_{i j}\right)$ is the cut of order $r$ of $\left(S_{i, i-j}, \mathfrak{S}_{i, i-j+2, i-j+1}\right)$.

(6.11) $H\left(i, i-j+1, \alpha_{k}\right)=\bigcup\left\{H\left(i, i-j+1, \alpha_{i-j+1}\right) ; f_{i-j+1, k}\left(\alpha_{i-j+1}\right)=\alpha_{k}\right\}$ if $k<i-j+1$, and $H\left(i, i-j+1, \alpha_{k}\right)=h_{i j}^{-1}\left(H\left(i, i-j+2, \alpha_{k}\right)\right) \cap H\left(i, i-j+1, f_{k, i-j+1}\left(\alpha_{k}\right)\right)$ if $k>i-j+1$. Then the sequence $\mathfrak{H}_{i . i-j+1, k}, k=1,2, \cdots$, satisfies the following conditions.

(6.12) For any point $p$ of $S_{i j}$ and any neighborhood $U$ of $p$, there exists $k$ such that $\operatorname{Star}\left(p, \mathfrak{S}_{i, i-j+1, k}\right) \subset U$.

(6.13) For any $k$ and any $\alpha_{k} \in A_{k}, \quad H\left(i, i-j+1, \alpha_{k}\right)=\mathbf{U}\left\{H\left(i, i-j+1, \alpha_{k+1}\right)\right.$; $\left.f_{k+1, k}\left(\alpha_{k+1}\right)=\alpha_{k}\right\}$.

(6.14) For any $k$ with $k \geqq i-j+1$ and any $\alpha_{k} \in A_{k}, h_{i j} \mid H\left(i, i-j+1, \alpha_{k}\right)$ is a homeomorphism of $H\left(i, i-j+1, \alpha_{k}\right)$ onto $H\left(i, i-j+2, \alpha_{k}\right)$.

iv) Let $h_{i}=h_{i 1} h_{i 2} \cdots h_{i i}$; then $h_{i}$ is a closed continuous mapping of $S_{i i}$ onto $R_{0}$ such that $h_{i} \mid H\left(i, i, \alpha_{i}\right)$ is a homeomorphism of $H\left(i, 1, \alpha_{i}\right)$ onto $F\left(0, \alpha_{i}\right)$ for every $\alpha_{i} \in A_{i}$. Let $s=s(x), x \in R_{0}$, be the greatest integer such that order $\left(x, \widetilde{\mho}_{0 s}\right) \leqq r$. When $\operatorname{order}\left(x, \widetilde{F}_{01}\right)>r$, let $s(x)=0$.

First we consider the case $s(x)=0$. Then $\operatorname{order}\left(x, \widetilde{F}_{0 i}\right)>r$ for every $i$. Hence $\operatorname{order}\left(x, h_{i 1} h_{i 2} \cdots h_{i j}\right)=1$ for every $j$. Especially $\operatorname{order}\left(x, h_{i}\right)=1$.

Next we consider the case $0<s(x) \leqq i$. Then $\operatorname{order}\left(x, h_{i 1} \cdots h_{i, i-s}\right)^{20)}=1$ and $\operatorname{order}\left(x, h_{i 1} \cdots h_{i, i-s+1}\right)=\operatorname{order}\left(x, \mathfrak{F}_{0 s}\right)$. Let $y$ be an arbitrary point of $\left(h_{i 1} \cdots h_{i, i-s+1}\right)^{-1}$ $(x)$ and then $\operatorname{order}\left(y, \mathfrak{H}_{i s s}\right)=1$ by Remark 5.4. Hence $\operatorname{order}\left(y, h_{i, i-s+2} \cdots h_{i i}\right)=1$. Therefore $\operatorname{order}\left(x, h_{i}\right)=\operatorname{order}\left(x, \mathfrak{F}_{0 s}\right)$.

Last we consider the case $i<s(x)$. Then $\operatorname{order}\left(x, \mathfrak{F}_{0 i}\right) \leqq \operatorname{order}\left(x, \mathfrak{F}_{0 s}\right) \leqq r$. Hence $\operatorname{order}\left(x, h_{i 1}\right)=\operatorname{order}\left(x, \mathfrak{F}_{0 i}\right)$. By the same argument as in the case of $0<s(x) \leqq i$ we get $\operatorname{order}\left(x, h_{i}\right)=\operatorname{order}\left(x, \widetilde{\mho}_{0 i}\right)$.

In any case of the above, we get $\operatorname{order}\left(x, h_{i}\right) \leqq r$ and hence (6.15) order $h_{i} \leqq r$.

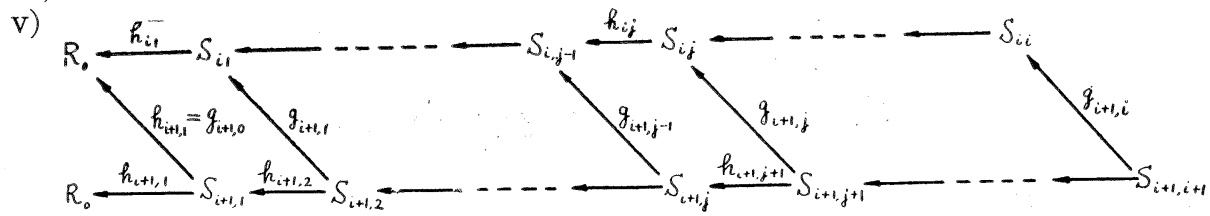

Diagram 2.

20) When $s(x)=i$, there is no necessity for considering this. 
Let $h_{i+1,1}=g_{i+1,0}$. Since $g_{i+1,0}\left(H\left(i+1, i+1, \alpha_{i}\right)\right)=F\left(0, \alpha_{i}\right)$ for every $\alpha_{i} \in A_{i}$, there exists $g_{i+1,1}: S_{i+1,2} \rightarrow S_{i 1}$ with $g_{i+1,0} h_{i+1,2}=h_{i 1} g_{i+1,1}$ by Lemma 5.5. By the successive applications of Lemma 5.5 we get $g_{i+1, j}: S_{i+1, j+1} \rightarrow S_{i j}$ with

(6.16) $g_{i+1, j-1} h_{i+1, j+1}=h_{i j} g_{i+1, j}, j=0,1, \cdots, i$,

since $g_{i+1, j+1}\left(H\left(i+1, i+1-(j-1), \alpha_{i-(j-1)}\right)=H\left(i, i-(j-1)+1, \alpha_{i-j+1}\right)^{21)}\right.$ for every $\alpha_{i-j+1} \in A_{i-j+1}$. Therefore the equality $h_{i 1} h_{i 2} \cdots h_{i i} g_{i+1, i}=g_{i+1,0} h_{i+1,2} h_{i+1,3} \cdots h_{i+1, i+1}$ holds. Since $g_{i+1,0}=h_{i+1,1}$, we get

(6.17) $h_{i} g_{i+1, i}=h_{i+1}$.

Therefore

(6.18) $g_{10} g_{21} \cdots g_{i, i-1}=h_{i}$.

Let $\alpha_{i+1}$ be an arbitrary element of $A_{i+1}$ and $\alpha_{i}=f_{i+1, i}\left(\alpha_{i+1}\right)$. Let $h_{i+1,2, \alpha_{i}}=$ $h_{i+1,2}\left|H\left(i+1, i, \alpha_{i}\right), g_{i+1,0, \alpha_{i}}=g_{i+1,0}\right| h_{i+1,2}\left(H\left(i+1, i, \alpha_{i}\right)\right)=g_{i+1,0} \mid H\left(i+1, i+1, \alpha_{i}\right)$ and $h_{i, 1, \alpha_{i}}=h_{i 1} \mid H\left(i, i, \alpha_{i}\right)$. Then by the construction of $g_{i+1,1}$ (cf. the proof of Lemma 5.5) $g_{i+1,1} \mid H\left(i+1, i, \alpha_{i}\right)=h_{i, 1, \alpha_{i}}^{-1} g_{i+1,0, \alpha_{i}} h_{i+1,2, \alpha_{i}}$. Since $h_{i+1,2}\left(H\left(i+1, i, \alpha_{i+1}\right)\right)=H(i+1$, $\left.i+1, \alpha_{i+1}\right)$ and $h_{i 1}\left(H\left(i, i, \alpha_{i+1}\right)\right)=F\left(0, \alpha_{i+1}\right)=g_{i+1,0}\left(H\left(i+1, i+1, \alpha_{i+1}\right)\right)$, we get $g_{i+1,1}\left|H\left(i+1, i, \alpha_{i+1}\right)=h_{i, 1, \alpha_{i}}^{-1} g_{i+1,0, \alpha_{i}} h_{i+1,2, \alpha_{i}}\right| H\left(i+1, i, \alpha_{i+1}\right)$. Thus $g_{i+1,1} \mid H(i+1, i$, $\left.\alpha_{i+1}\right)$ is a homeomorphism of $H\left(i+1, i, \alpha_{i+1}\right)$ onto $H\left(i, i, \alpha_{i+1}\right)$. By the same argument as above we can know succeedingly that for every $\alpha_{i+1} \in A_{i+1} g_{i+1, j} \mid H(i+1$, $\left.i-j+1, \alpha_{i+1}\right)$ is a homeomorphism of $H\left(i+1, i-j+1, \alpha_{i+1}\right)$ onto $H\left(i, i-j+1, \alpha_{i+1}\right)$, $j=1, \cdots, i$. Especially $g_{i+1, i} \mid H\left(i+1,1, \alpha_{i+1}\right)$ is a homeomorphism of $H(i+1,1$, $\left.\alpha_{i+1}\right)$ onto $H\left(i, 1, \alpha_{i+1}\right)$ for every $\alpha_{i+1} \in A_{i+1}$.

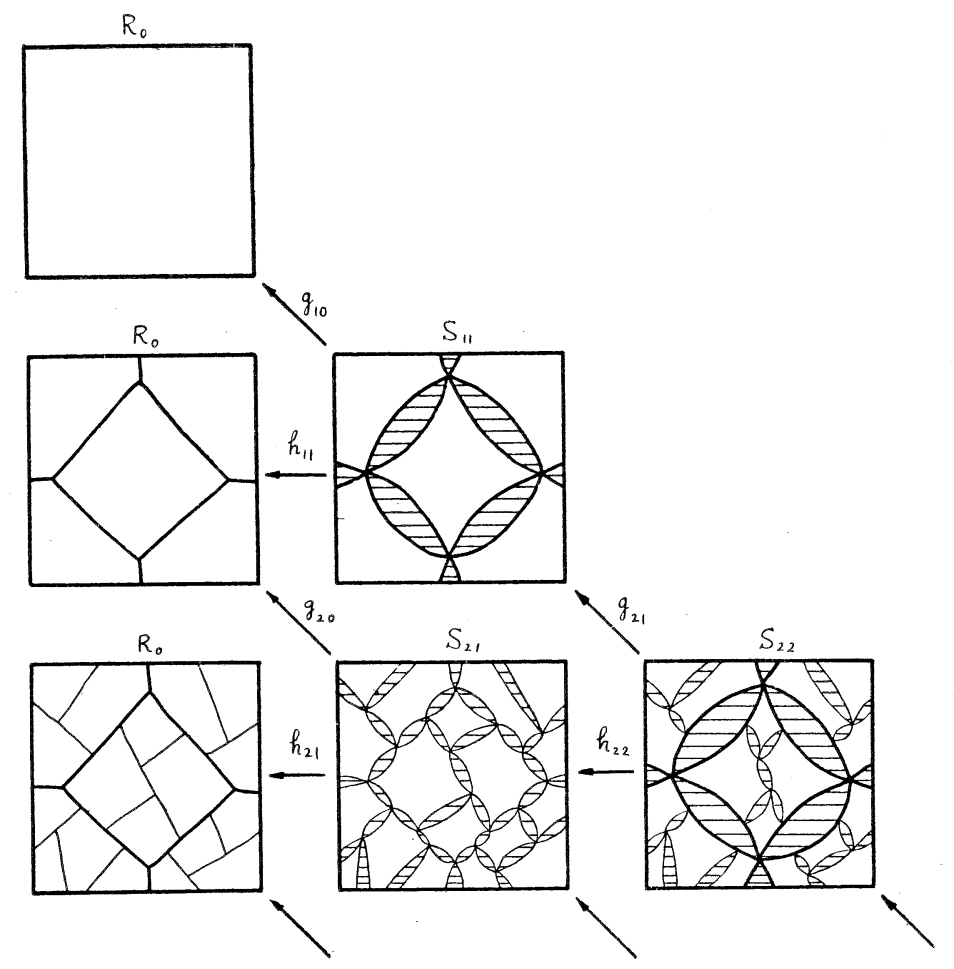

Figure 1.

21) More precisely we have to prove this equality as well as (6.16) by the induction on $j$. The proof is rather simple and is left to the reader. 
vi) Let $R_{i}=S_{i i}, F\left(i, \alpha_{j}\right)=H\left(i, 1, \alpha_{j}\right)$ and $\widetilde{\mho}_{i j}=\left\{F\left(i, \alpha_{j}\right) ; \alpha_{j} \in A_{j}\right\}, i=1,2, \cdots$, $j=1,2, \cdots$. Let $T=\lim \left\{R_{i}, g_{i+1, i}(i=0,1,2, \cdots)\right\}$ and $\pi_{i}$ the projection of $T$ onto $R_{i}$. Since every $R_{i}$ is metrizable by Remark 5.3, $T$ is also metrizable. Let $K\left(\alpha_{i}\right)=\pi_{i}^{-1}\left(F\left(i, \alpha_{i}\right)\right)$; then it can easily be seen from iii) and v) that $\Omega_{i}=$ $\left\{K\left(\alpha_{i}\right) ; \alpha_{i} \in A_{i}\right\}, i=1,2, \cdots$, is a sequence of locally finite closed coverings of $T$ such that

(6.19) $\Re_{i+1}$ refines $\Re_{i}$ for every $i$,

(6.20) for any point $\xi$ of $T$ and neighborhood $U$ of $\xi$ there exists $i$ with $\operatorname{Star}\left(\xi, \Re_{i}\right) \subset U$.

vii) The projection $\pi_{0}: T \rightarrow R_{0}$ is clearly continuous. To prove order $\pi_{0} \leqq r=$ $n-m+1$, let us place the assumption that order $\pi_{0}>r$. Then there exist $r+1$ points $\xi_{1}, \cdots, \xi_{r+1}$ of $T$ with $\pi_{0}\left(\xi_{j}\right)=\pi_{0}\left(\xi_{1}\right), j=2, \cdots, r+1$. Since $\xi_{j}, j=1, \cdots$, $r+1$, are mutually different, there exists $i$ such that $\pi_{i}\left(\xi_{j}\right), j=1, \cdots, r+1$, are mutually different points of $R_{i}$. Since $g_{10} g_{21} \cdots g_{i, i-1} \pi_{i}\left(\xi_{j}\right)=\pi_{0}\left(\xi_{1}\right), j=1, \cdots, r+1$, we get $h_{i} \pi_{i}\left(\xi_{j}\right)=\pi_{0}\left(\xi_{1}\right), j=1, \cdots, r+1$, by (6.18). Thus we get order $h_{i}>r$. On the other hand order $h_{i} \leqq r$ by (6.15), which is a contradiction. Therefore we get order $\pi_{0} \leqq r$.

viii) Now let us prove the closedness of $\pi_{0}$. Let $i$ be an arbitrary positive integer and $\alpha_{i}$ an arbitrary element of $A_{i}$. Since a) $\pi_{i}\left(K\left(\alpha_{i}\right)\right)=\pi_{i}\left(\pi_{i}^{-1}\left(F\left(i, \alpha_{i}\right)\right)\right)=$ $F\left(i, \alpha_{i}\right)$, b) $h_{i}\left(F\left(i, \alpha_{i}\right)\right)=h_{i}\left(H\left(i, 1, \alpha_{i}\right)\right)=F\left(0, \alpha_{i}\right)$ by iv) and c) $h_{i}=g_{10} \cdots g_{i, i-1}$ by (6.18), we get $\pi_{0}\left(K\left(\alpha_{i}\right)\right)=g_{10} \cdots g_{i, i-1} \pi_{i}\left(K\left(\alpha_{i}\right)\right)=h_{i}\left(F\left(i, \alpha_{i}\right)\right)=F\left(0, \alpha_{i}\right)$. Let $F$ be a non-empty closed subset of $T$ and $x_{0}$ an arbitrary point of $\overline{\pi_{0}(F)}$. Then $\pi_{0}\left(F \cap K\left(\alpha_{1}\right)\right) \subset F\left(0, \alpha_{1}\right)$ and hence $\left.\overline{\pi_{0}(F)}=\bigcup\left\{\overline{\pi_{0}\left(F \cap K\left(\alpha_{1}\right)\right.}\right) ; \alpha_{1} \in A_{1}\right\}$ by the local finiteness of $\mathfrak{F}_{01}=\left\{F\left(0, \alpha_{1}\right) ; \alpha_{1} \in A_{1}\right\}$. Therefore there exists $\alpha_{1}\left(x_{0}\right) \in A_{1}$ such that $x_{0} \in \overline{\pi_{0}\left(F \cap K\left(\alpha_{1}\left(x_{0}\right)\right)\right)}$. By (6.19) and the local finiteness of $\mathfrak{F}_{02}, \overline{\pi_{0}\left(F \cap K\left(\alpha_{1}\left(x_{0}\right)\right)\right)}=$ U $\left.\left.\left\{\overline{\pi_{0}\left(F \cap K\left(\alpha_{1}\left(x_{0}\right)\right) \cap K\left(\alpha_{2}\right)\right.}\right)=\overline{\pi_{0}\left(F \cap K\left(\alpha_{2}\right)\right.}\right) ; \alpha_{2} \in A_{2}, f_{21}\left(\alpha_{2}\right)=\alpha_{1}\left(x_{0}\right)\right\}$. Hence there exists $\alpha_{2}\left(x_{0}\right) \in A_{2}$ with $f_{21}\left(\alpha_{2}\left(x_{0}\right)\right)=\alpha_{1}\left(x_{0}\right)$ such that $x_{0} \in \overline{\pi_{0}\left(F \cap K\left(\alpha_{2}\left(x_{0}\right)\right)\right)}$. Repeating this argument we get $\alpha_{i}\left(x_{0}\right) \in A_{i}$ with $f_{i, i-1}\left(\alpha_{i}\left(x_{0}\right)\right)=\alpha_{i-1}\left(x_{0}\right), i=2,3, \cdots$, such that $x_{0} \in \overline{\pi_{0}\left(F \cap K\left(\alpha_{i}\left(x_{0}\right)\right)\right)}$.

Let $r_{1}=\operatorname{order}\left(x_{0}, \pi_{0}\right)$ and $\left\{\xi_{1}, \cdots, \xi_{r_{1}}\right\}=\pi_{0}^{-1}\left(x_{0}\right)$. By (6.20) there exists $i_{0}$ such that $\operatorname{Star}\left(\xi_{i}, \mathfrak{R}_{i_{0}}\right) \cap \operatorname{Star}\left(\xi_{j}, \Re_{i_{0}}\right)=\phi$ whenever $i \neq j$. Then evidently $\pi_{i_{0}}\left(\xi_{i}\right), i=$ $1, \cdots, r_{1}$, are mutually different points of $R_{i_{0}}$ such that $\operatorname{Star}\left(\pi_{i_{0}}\left(\xi_{i}\right), \mho_{i_{0}, i_{0}}\right) \cap$ $\operatorname{Star}\left(\pi_{i_{0}}\left(\xi_{j}\right), \widetilde{F}_{i_{0}, i_{0}}\right)=\phi$ whenever $i \neq j$. Since a) $x_{0} \in \overline{\pi_{0}\left(F \cap K\left(\alpha_{i_{0}}\left(x_{0}\right)\right)\right)} \subset \pi_{0}\left(K\left(\alpha_{i_{0}}\left(x_{0}\right)\right)\right)=$ $F\left(0, \alpha_{i_{0}}\left(x_{0}\right)\right)$, b) $\pi_{0}\left(K\left(\alpha_{i_{0}}\left(x_{0}\right)\right)\right)=h_{i_{0}} \pi_{i_{0}}\left(K\left(\alpha_{i_{0}}\left(x_{0}\right)\right)\right)=h_{i_{0}}\left(F\left(i_{0}, \alpha_{i_{0}}\left(x_{0}\right)\right)\right)$, and c) $h_{i_{0}}^{-1}\left(x_{0}\right)=$ $\left\{\pi_{i_{0}}\left(\xi_{i}\right) ; i=1, \cdots, r_{1}\right\}$, there exists one and only one $\xi_{i}$ such that $\pi_{i_{0}}\left(\xi_{i}\right) \in F\left(i_{0}, \alpha_{i_{0}}\left(x_{0}\right)\right)$. We can assume without loss of generality that $\xi_{1}=\xi_{i}$. Since order $\left(\pi_{i_{0}}\left(\xi_{1}\right)\right.$, $\left.g_{i_{0}+1, i_{0}} g_{i_{0}+2, i_{0}+1} \cdots g_{j, j-1}\right)=1$ whenever $j>i_{0}$, we get $\pi_{i_{0}}^{-1} \pi_{i_{0}}\left(\xi_{1}\right)=\xi_{1}$. Hence $\xi_{1} \in \pi_{i_{0}}^{-1}\left(F\left(i_{0}\right.\right.$, $\left.\left.\alpha_{i_{0}}\left(x_{0}\right)\right)\right)=K\left(\alpha_{i_{0}}\left(x_{0}\right)\right)$. In other words $\pi_{0}^{-1}\left(x_{0}\right) \cap K\left(\alpha_{i_{0}}\left(x_{0}\right)\right)=\xi_{1}$. When $j>i_{0}, K\left(\alpha_{j}\left(x_{0}\right)\right) \subset$ $K\left(\alpha_{i n}\left(x_{0}\right)\right)$ and hence $\pi_{0}^{-1}\left(x_{0}\right) \cap K\left(\alpha_{j}\left(x_{0}\right)\right)=\xi_{1}$ by $x_{0} \in \pi_{0}\left(K\left(\alpha_{j}\left(x_{0}\right)\right)\right)$. Thus $\xi_{1} \in \bigcap_{i=1}^{\infty} K\left(\alpha_{i}\left(x_{0}\right)\right)$ and by $(6.20) \quad \xi_{1}=\bigcap_{i=1}^{\infty} K\left(\alpha_{i}\left(x_{0}\right)\right)$. Let $U$ be an arbitrary neighborhood of $\xi_{1}$ and there exists $i_{1}$ such that $\operatorname{Star}\left(\xi_{1}, \Re_{i_{1}}\right) \subset U$. Since $U \cap F \supset$

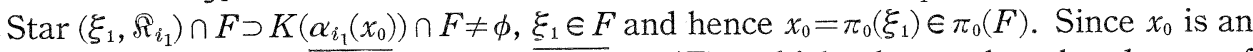
arbitrary point of $\overline{\pi_{0}(F)}$, we get $\overline{\pi_{0}(F)} \subset \pi_{0}(F)$, which shows the closedness of $\pi_{0}(F)$. Thus the closedness of $\pi_{0}$ is proved.

ix) Finally let us show $\operatorname{dim} T=m$. We have already known in vii) and viii) that $\pi_{0}$ is a closed continuous mapping of $T$ onto $R_{0}$ with order $\pi_{0} \leqq r$. Hence Morita [18, Theorem 4] can be applied and $\operatorname{dim} T \geqq \operatorname{dim} R_{0}-r+1=n-$ $(n-m+1)+1=m$. 
Now we proceed to prove $\operatorname{dim} T \leqq m$. Let $i$ be an arbitrary positive integer and $x$ an arbitrary point of $R_{i}$. If $\operatorname{order}\left(x, \mathfrak{F}_{i i}\right)>1$ there exist different indices $\alpha_{i}$ and $\beta_{i}$ of $A_{i}$ such that $x \in F\left(i, \alpha_{i}\right) \cap F\left(i, \beta_{i}\right)$. Then $h_{i 2} h_{i 3} \cdots h_{i i}(x) \in h_{i 2} \cdots h_{i i}$ $\left.\left(F\left(i, \alpha_{i}\right) \cap F\left(i, \beta_{i}\right)\right) \subset h_{i 2} \cdots h_{i i}\left(F\left(i, \alpha_{i}\right)\right) \cap h_{i 2} \cdots h_{i i} F\left(i, \beta_{i}\right)\right)=H\left(i, i, \alpha_{i}\right) \cap H\left(i, i, \beta_{i}\right)$ by (6.14). Since for every point $y \in S_{i 1}$ either $\operatorname{order}\left(y, \mathfrak{S}_{i i i}\right)=1$ or $>r$ holds, we get order $\left(h_{i 2} \cdots h_{i i}(x), \mathfrak{S}_{i i i}\right)>r$. Hence order $\left(h_{i}(x), \mathfrak{F}_{0 i}\right)>r$.

Let

(6.21) $P_{i}=\bigcup\left\{\bigcap_{j=1}^{r+1} F(0, \alpha(j)) ; \alpha(1), \cdots, \alpha(r+1)\right.$ are mutually different indices of $\left.A_{i}\right\}$;

then by (6.4) and the local finiteness of $\widetilde{F}_{0 i}, P_{i}$ is a closed subset of $R_{0}$ with $\operatorname{dim} P_{i} \leqq n-(r+1)+1=n-(n-m+1)=m-1$ (cf. Morita [15, Theorem 3.2]). Let $\alpha_{i}$ be an arbitrary element of $A_{i}$ and

(6.22) $Q_{i}=\left\{x ; x \in R_{i}\right.$, $\left.\operatorname{order}\left(x, \mathfrak{\mho}_{i i}\right)>1\right\}$

and then $Q_{i}$ is a closed subset of $R_{i}$ by the local finiteness of $\varlimsup_{i i}$ and $P_{i} \cap$ $F\left(0, \alpha_{i}\right) \supset h_{i}\left(Q_{i} \cap F\left(i, \alpha_{i}\right)\right)$. Since $h_{i} \mid F\left(i, \alpha_{i}\right)$ is a homeomorphism, we get $\operatorname{dim} Q_{i} \cap$ $F\left(i, \alpha_{i}\right) \leqq m-1$. Then by the local finiteness of $\mathfrak{\mho}_{i i}$ we get

(6.23) $\operatorname{dim} Q_{i} \leqq m-1$.

Let $x$ be an arbitrary point of $Q_{i}$ and $\gamma_{i}$ and $\delta_{i}$ be elements of $A_{i}$ with $\gamma_{i} \neq \delta_{i}$ such that $x \in F\left(i, \gamma_{i}\right) \cap F\left(i, \delta_{i}\right)$. Let $s=s\left(h_{i}(x)\right)$, where $s$ is the function of $R_{0}$ defined in iv). Let us consider three cases. a) When $s=0$, we get $\operatorname{order}\left(h_{i}(x)\right.$, $\left.h_{i+1}\right)=1$ by iv). Since $h_{i} g_{i+1, i}=h_{i+1}$ by $(6.17), g_{i+1, i}^{-1}(x)$ is a single point. Since $g_{i+1, i}\left(F\left(i+1, \alpha_{i}\right)\right)=F\left(i, \alpha_{i}\right)$ for every $\alpha_{i} \in A_{i}$, we get $g_{i+1, i}^{-1}(x) \in F\left(i+1, \gamma_{i}\right) \cap$ $F\left(i+1, \delta_{i}\right)$. b) When $s \geqq i$, then by iv) $\operatorname{order}\left(h_{i}(x), h_{i}\right)=\operatorname{order}\left(h_{i}(x), \widetilde{\mho}_{0 i}\right) \leqq \operatorname{order}\left(h_{i}(x)\right.$, $\left.\mathfrak{F}_{0 s}\right) \leqq r$. Then by the construction of $R_{i}$ and Remark 5.4 , we get order $\left(x, \mathfrak{\mho}_{i i}\right)=$ 1 , which is a contradiction. Therefore the case $s \geqq i$ does not happen. c) When $s<i$, we have already known in iv) that $\operatorname{order}\left(h_{i}(x), h_{i}\right)=\operatorname{order}\left(h_{i}(x), h_{i+1}\right)=$ $\operatorname{order}\left(h_{i}(x), \mathfrak{F}_{0 s}\right)$. Hence $g_{i+1, i} \mid h_{i+1}^{-1}\left(h_{i}(x)\right)$ is a homeomorphism of $h_{i+1}^{-1}\left(h_{i}(x)\right)$ onto $h_{i}^{-1}\left(h_{i}(x)\right)$ by the relation (6.16). Since $\left|h_{i+1}^{-1}\left(h_{i}(x)\right)\right|=\left|h_{i}^{-1}\left(h_{i}(x)\right)\right|, g_{i+1, i}^{-1}(x)$ is a single point. Therefore $g_{i+1, i}^{-1}(x) \in F\left(i+1, \gamma_{i}\right) \cap F\left(i+1, \delta_{i}\right)$.

Thus we can conclude that for every $i g_{i+1, i}^{-1}\left(Q_{i}\right) \subset L_{i+1}=\left\{x ; x \in R_{i+1}\right.$, $\left.\operatorname{order}\left(x, \widetilde{\mho}_{i+1, i}\right)>1\right\}$. Since evidently $g_{i+1, i}\left(L_{i+1}\right) \subset Q_{i}$, we get $g_{i+1, i}^{-1}\left(Q_{i}\right) \subset L_{i+1}$ and hence $g_{i+1, i} \mid L_{i+1}$ is a homeomorphism of $L_{i+1}$ onto $Q_{i}$. Since $L_{j} \subset Q_{j}$ for every $j, g_{i+1, i} \cdots g_{i+j, i+j-1} \mid\left(g_{i+1, i} \cdots g_{i+j, i+j-1}\right)^{-1}\left(Q_{i}\right)$ is a homeomorphism of $\left(g_{i+1, i} \cdots\right.$ $\left.g_{i+j, i+j-1}\right)^{-1}\left(Q_{i}\right)$ onto $Q_{i}$ for every positive integer $j$. Therefore

(6.24) $K_{i}=\left\{\xi ; \xi \in T\right.$, order $\left.\left(\xi, \Re_{i}\right)>1\right\}$

is homeomorphic to $Q_{i}$ and hence by (6.23) we get

(6.25) $\operatorname{dim} K_{i} \leqq m-1$.

Let

(6.26) $\quad T_{0}=T-\bigcup_{i=1}^{\infty} K_{i}$

and

(6.27) $U\left(\alpha_{i}\right)=T_{0} \cap K\left(\alpha_{i}\right), \alpha_{i} \in A_{i}$,

and then $\mathfrak{H}_{i}=\left\{U\left(\alpha_{i}\right) ; \alpha_{i} \in A_{i}\right\}, i=1,2, \cdots$, is as can easily be seen a sequence of locally finite closed coverings of $T_{0}$ which satisfies the following conditions.

(6.28) $\mathfrak{U}_{i+1}$ refines $\mathfrak{U}_{i}$ for every $i$.

(6.29) order $\mathfrak{H}_{i} \leqq 1$ for every $i$.

(6.30) For any point $\xi_{0}$ of $T_{0}$ and any neighborhood $G_{0}$ of $\xi_{0}$ there exists $i$ such that $\operatorname{Star}\left(\xi_{0}, \mathfrak{U}_{i}\right) \subset G_{0}$.

Therefore by Theorem 3.2 we get 
(6.31) $\operatorname{dim} T_{0} \leqq 0$.

Since $K_{i}$ is closed in $T$ and $\operatorname{dim} K_{i} \leqq m-1$ by (6.25) we get by the sum theorem (6.32) $\operatorname{dim} \bigcup_{i=1}^{\infty} K_{i} \leqq m-1$.

Hence by Theorem $3.2 T$ is the sum of $m+1$ subspaces $T_{i}, i=0,1, \cdots, m$, with $\operatorname{dim} T_{i} \leqq 0$ and hence we get at last

(6.33) $\operatorname{dim} T \leqq m$.

Thus our construction is completed.

Concerning the dimension of an inverse limiting space we get the following theorem.

Theorem 6.1. Let $R=\lim \left\{R_{i}, f_{i j}\right\}$ where $R_{i}$ is a metric space and $f_{i j}: R_{i} \rightarrow R_{j}$ $(i>j)$ is continuous, for every $i$. Then $\operatorname{dim} R \leqq \lim \inf \operatorname{dim} R_{i}$.

Proof. When $\lim \inf \operatorname{dim} R_{i}=\infty$, the theorem is evidently true. When $\lim \inf \operatorname{dim} R_{i}=n(<\infty), R$ is homeomorphic to $\lim \left\{R_{i^{\prime}} ; f_{i^{\prime} j^{\prime}}\right\}$ where $\left\{R_{i^{\prime}}, f_{i^{\prime} j^{\prime}}\right\}$ is a cofinal subsystem of $\left\{R_{i}, f_{i j}\right\}$ and $\operatorname{dim} R_{i^{\prime}} \leqq n$ for every $i^{\prime}$. Hence we can assume with no loss of generality that $\operatorname{dim} R_{i} \leqq n$ for every $i$. It is easy to construct by the induction a sequence of open coverings $\mathfrak{H}_{j i}, i=1,2, \cdots$, of $R_{j}$, $j=1,2, \cdots$, which satisfies the following conditions.

(6.34) mesh $\mathfrak{H}_{j i}<1 / i$ for every $j$ and $i$.

(6.35) $\mathfrak{u}_{j, i+1}$ refines $\mathfrak{H}_{j i}$ for every $j$ and $i$.

(6.36) $\mathfrak{u}_{j i}$ refines $f_{j, j-1}^{-1}\left(\mathfrak{H}_{j-1, i}\right)=\left\{f_{j, j-1}^{-1}(U) ; U \in \mathfrak{H}_{j-1, i}\right\}$ for every $j$ and $i$.

It is also easy to construct by the induction a locally finite open covering $\mathfrak{B}_{i}$ of $R_{i}, i=1,2, \cdots$, which satisfies the following conditions.

(6.37) $\overline{\mathfrak{V}}_{i+1}=\left\{\bar{V} ; V \in \mathfrak{B}_{i+1}\right\}$ refines $\mathfrak{U}_{i+1, i+1}$ as well as $f_{i+1, i}^{-1}\left(\mathfrak{V}_{i}\right)$.

(6.38) order $\overline{\aleph_{i}} \leqq n+1$.

Let

(6.39) $\widetilde{\mho}_{i}=\left\{\pi_{i}^{-1}(\bar{V}) ; V \in \mathfrak{V}_{i}\right\}, i=1,2, \cdots$,

where $\pi_{i}: R \rightarrow R_{i}$ is the projection. Then $\widetilde{\mho}_{i}, i=1,2, \cdots$, is a sequence of locally finite closed coverings of $R$ such that

(6.40) $\widetilde{\mho}_{i+1}$ refines $\widetilde{\mho}_{i}$ for every $i$,

(6.41) order $\mathfrak{\mho}_{i} \leqq n+1$ for every $i$.

Let $\xi$ be an arbitrary point of $R$ and $W$ an arbitrary neighborhood of $\xi$ and then there exist $i$ and a neighborhood $W_{i}$ of $\pi_{i}(\xi)$ such that $\pi_{i}^{-1}\left(W_{i}\right) \subset W$. Then by (6.34) there exists $j$ such that $\operatorname{Star}\left(\pi_{i}(\xi), \mathfrak{u}_{i j}\right) \subset W_{i}$. By (6.35) we can assume with no loss of generality that $i \leqq j$. By (6.36) and (6.37) we get (6.42) $\operatorname{Star}\left(\xi, \mathfrak{F}_{j}\right) \subset \pi_{i}^{-1}\left(W_{i}\right) \subset W$.

Since $\mathfrak{F}_{i}, i=1,2, \cdots$, satisfy the conditions $(6.40),(6.41),(6.42)$, we get $\operatorname{dim} R \leqq n$ by Theorem 3.2. Thus the theorem is proved.

Remark 6.2. Even if every $f_{i j}$ in the above theorem is onto and $\operatorname{dim} R_{i}=n$ for every $i$, we cannot assert that $\operatorname{dim} R=n$. The space $T$ which has been constructed in this paragraph is such an example.

\section{$\S 7$. Characterization of dimension by means of a sequence of coverings}

The following lemma is a slight modification of Morita's metrization theorem [18, Theorem 1].

Lemma 7.1. In order that a topological space $R$ be metrizable it is necessary 
and sufficient that there exists a sequence of locally finite coverings $\widetilde{F}_{i}, i=1,2, \cdots$, of $R$ such that

(7.1) for any neighborhood $U$ of any point $x$ of $R$ there exists $i$ with $\operatorname{Star}\left(x, \overline{\mathfrak{F}}_{i}\right) \subset U$.

Now we proceed to get a' weaker condition which characterizes dimension than that of $(B)$ of $\S 3$.

THeOREM 7.2. In order that a topological space $R$ be a metrizable space with $\operatorname{dim} R \leqq n$ it is necessary and sufficient that there exists a sequence of locally finite coverings $\widetilde{\gamma}_{i}=\left\{F_{\alpha_{i}} ; \alpha_{i} \in A_{i}\right\}, i=1,2, \cdots$, which satisfies the following conditions.

(7.2) $\widetilde{\mho}_{i+1}$ refines $\mathfrak{\mho}_{i}$ for every $i$.

(7.3) lim inf order $\left(x, \mathfrak{\mho}_{i}\right) \leqq n+1$ for every $x \in R$.

(7.4) For any point $x \in R$ and any neighborhood $U$ of $x$ there exists $i$ with $\operatorname{Star}\left(x, \widetilde{\mho}_{i}\right) \subset U$.

Proof. The necessity of the theorem is obvious from the implication $(\mathrm{A}) \rightarrow$ (B) of $\S 3$.

Let us assume the existence of a sequence satisfying the conditions of the proposition. Then $R$ is metrizable by Lemma 7.1. For every $i$ let us define a single-valued mapping $f_{i+1, i}$ of $A_{i+1}$ into $A_{i}$ in such a way that $f_{i+1, i}\left(\alpha_{i+1}\right)=\alpha_{i}$ yields $\bar{F}_{\alpha_{i+1}} \subset F_{\alpha_{i}}$.

Consider $A_{i}$ as a topological space with discrete topology, $i=1,2, \cdots$. Let $A_{0}$ be the aggregate of points $a=\left(\alpha_{1}, \alpha_{2}, \cdots\right)$ of $\lim \left\{A_{i}, f_{i+1, i}\right\}$ such that $\bigcap_{i=1}^{\infty} F_{\alpha_{i}} \neq \phi$ and $f$ a mapping of $A_{0}$ into $R$ defined by $f(a)=f\left(\left(\alpha_{1}, \alpha_{2}, \cdots\right)\right)=\bigcap_{i=1}^{\infty} F_{\alpha_{i}}$. Then we can verify by the same argument as in the proof of $(B) \rightarrow(C)$ of $\S 3$ that $f$ is a closed continuous mapping of $A_{0}$ onto $R$.

To prove order $f \leqq n+1$ assume that $\operatorname{order} f>n+1$. Then there exists a point $x_{0}$ of $R$ with $\operatorname{order}\left(x_{0}, f\right)>n+1$. Let $a_{i}, i=1, \cdots, n+2$, be mutually different points of $A_{0}$ with $f\left(a_{i}\right)=x_{0}$ for $i=1, \cdots, n+2$. Let $j$ be an integer such that $\pi_{j}\left(a_{i}\right), i=1, \cdots, n+2$, are mutually different points of $A_{j}$, where $\pi_{j}$ is the projection of $A_{0}$ into $A_{j}$. By the condition (7.3) there exists $k$ with $k \geqq j$ such that $\operatorname{order}\left(x_{0}, \mathfrak{\mho}_{k}\right) \leqq n+1$. Since $k \geqq j, \pi_{k}\left(a_{i}\right), i=1, \cdots, n+2$, are mutually different points of $A_{k}$. Hence order $\left(x_{0}, \mathfrak{F}_{k}\right)>n+1$, which is a contradiction. Thus order $f \leqq n+1$ is true. Evidently $\operatorname{dim} A_{0} \leqq 0$. Hence the condition (C) of $\S 3$ is satisfied and we get $\operatorname{dim} R \leqq n$. The proof is completed.

The following proposition is a trivial consequence of Theorem 7.2.

Corollary 7.3 (C. H. Dowker and W. Hurewicz [6]). A metric space $R$ has dimension $\leqq n$ if and only if there exists a sequence of locally finite open coverings $\mathfrak{H}_{i}, i=1,2, \cdots$, of $R$ which satisfies the following conditions.

(7.5) $\overline{\mathfrak{U}}_{i+1}$ refines $\mathfrak{U}_{i}$ for every $i$.

(7.6) order $\mathfrak{H}_{i} \leqq n+1$ for every $i$.

(7.7) $\operatorname{mesh} \mathfrak{H}_{i} \rightarrow 0$ as $i \rightarrow \infty$.

The following theorem of P. Alexandroff and P. Urysohn is well known.

Lemma 7.4. In order that a topological space $R$ be metrizable it is necessary and sufficient that there exists a sequence of open coverings $\mathfrak{H}_{i}, i=1,2, \cdots$, which satisfies the following conditions.

$(7.8) \mathfrak{u}_{i+1}^{*}$ refines $\mathfrak{u}_{i}$ for every $i .^{22)}$

22) $\mathfrak{U}_{j}^{*}=\left\{\operatorname{Star}\left(U, \mathfrak{u}_{j}\right) ; U \in \mathfrak{U}_{j}\right\}$. 
(7.9) For any point $x$ of $R$ and any neighborhood $U$ of $x$ there exists $i$ with $\operatorname{Star}\left(x, \mathfrak{H}_{i}\right) \subset U$.

Theorem 7.5. In order that a topological space $R$ be a metrizable space with $\operatorname{dim} R \leqq n$ it is necessary and sufficient that there exists a sequence of open coverings $\mathfrak{H}_{i}, i=1,2, \cdots$, of $R$ which satisfies the following conditions.

(7.10) $\mathfrak{H}_{i+1}^{*}$ refines $\mathfrak{H}_{i}$ for every $i$.

(7.11) lim inf order $\left(x, \mathfrak{u}_{i}\right) \leqq n+1$ for every point $x \in R$.

(7.12) For any point $x \in R$ and any neighborhood $U$ of $x$ there exists $i$ with $\operatorname{Star}\left(x, \mathfrak{H}_{i}\right) \subset U$.

Proof. Let $R$ be a metrizable space with $\operatorname{dim} R \leqq n$. Let $\mathfrak{B}_{i}, i=1,2, \cdots$, be a sequence of open coverings of $R$ with mesh $\mathfrak{B}_{i}{ }^{23)}<1 / i$. Let $\mathfrak{H}_{1}$ be an open covering of $R$ with order $\mathfrak{U}_{1} \leqq n+1$ such that $\mathfrak{U}_{1}$ refines $\mathfrak{B}_{1}$. Now make the induction assumption that there exist open coverings $\mathfrak{U}_{i}, i=1, \cdots, m$, of $R$ with mesh $\mathfrak{H}_{i}<1 / i$ such that $\mathfrak{H}_{1}>\mathfrak{U}_{2}{ }^{*}>\mathfrak{U}_{2}>\mathfrak{H}_{3}{ }^{*}>\cdots>\mathfrak{H}_{m}{ }^{*}$ and order $\mathfrak{U}_{i} \leqq n+1, i=1, \cdots, m$. Let $\mathfrak{H}_{m+1}$ be an open covering of $R$ with order $\mathfrak{H}_{m+1} \leqq n+1$ such that $U_{m+1}^{*}$ refines $\mathfrak{H}_{m}$ as well as $\mathfrak{B}_{m+1}$. Thus there exists by the induction a sequence of open coverings $\mathfrak{H}_{i}, i=1,2, \cdots$, of $R$ such that a) mesh $\mathfrak{u}_{i}<1 / i$ for every $\left.i, b\right)$ order $\mathfrak{u}_{i} \leqq$ $n+1$ for every $i$, and c) $\mathfrak{l}_{i+1}^{*}$ refines $\mathfrak{u}_{i}$ for every $i$. Then the conditions (7.10), (7.11) and (7.12) are evidently satisfied.

Conversely let us assume the existence of a sequence of open coverings $\mathfrak{H}_{i}=\left\{U_{\alpha} ; \alpha \in A_{i}\right\}, i=1,2, \cdots$, satisfying the conditions of the proposition. Then $R$ is metrizable by Lemma 7.4. We shall prove $\operatorname{dim} R \leqq \mathrm{n}$. Define a singlevalued mapping $f_{i+1, i}: A_{i+1} \rightarrow A_{i}, i=1,2, \cdots$, in such a way that $f_{i+1, i}\left(\alpha_{i+1}\right)=\alpha_{i}$ yields $\operatorname{Star}\left(U_{a_{i+1}}, \mathfrak{H}_{i+1}\right) \subset U_{\alpha_{i}}$. For any $i$ and $j$ with $i>j$ let $f_{i j}=f_{j+1, j} \cdots f_{i-1, i-2} f_{i, i-1}$. Consider $A_{i}$ as a topological space with discrete topology. Let $R_{0}$ be the aggregate of points $a$ of $\lim \left\{A_{i}, f_{i j}\right\}$ with $\bigcap_{i=1}^{\infty} U_{\pi_{i}(a)} \neq \phi$, where $\pi_{j}, j=1,2, \cdots$, is the projection of $\lim A_{i}$ into $A_{j}$. When $\bigcap_{i=1}^{\infty} U_{\pi_{i}(\alpha)} \neq \phi$, it consists of one and only one point by the condition (7.12).

Let $f: R_{0} \rightarrow R$ be a mapping such that $f(a)=\bigcap_{i=1}^{\infty} U_{\pi_{i}(a)}$. Let us show that $f$ is onto. Let $x$ be an arbitrary point of $R$; then by the condition (7.11) there exists a sequence of positive integers $m_{i}, i=1,2, \cdots$, with $m_{1}<m_{2}<\cdots$ such that $\operatorname{order}\left(x, \mathfrak{H}_{m_{i}}\right) \leqq n+1$ for every $i$. Let $B_{m_{i}}=\left\{\alpha ; \alpha \in A_{m_{i}}, x \in U_{\alpha}\right\}$; then $B_{m_{i}}, i=$ $1,2, \cdots$, is a finite set such that $f_{m_{i+1} m_{i}}\left(B_{m_{i+1}}\right) \subset B_{m_{i}}$ holds for every $i$. Since an inverse limiting space of non-empty compact Hausdorff spaces is not empty, we get $\lim \left\{B_{m_{i}}, f_{m_{i+1} m_{i}}\right\} \neq \phi$. Let $b$ be an arbitrary point of $\lim B_{m_{i}}$ and $a$ a point of $\lim \left\{A_{i}, f_{i j}\right\}$ such that $\pi_{m_{i}}(b)=\pi_{m_{i}}(a)$ for every $i$. Since $\bigcap_{i=1}^{\infty} U_{\pi_{i}(a)}=$ $\bigcap_{i=1}^{\infty} U_{\pi_{m_{i}}(b)}=x, a$ is a point of $R_{0}$ such that $f(a)=x$. Thus $f$ is onto. Let

(7.13) $\widetilde{\mho}_{i}=\left\{F_{\alpha_{i}}=f\left(\pi_{i}^{-1}\left(\alpha_{i}\right) \cap R_{0}\right) ; \alpha_{i} \in A_{i}\right\}$;

then $\widetilde{\mho}_{i}$ is a covering of $R$ since $f$ is onto. Let us prove that $\widetilde{\mho}_{i}$ is locally finite. Let $x$ be an arbitrary point of $R$ and there exist positive integers $m_{j}(x)$, $j=1,2, \cdots$, such that $m_{1}(x)<m_{2}(x)<\cdots$ and order $\left(x, \mathfrak{U}_{m_{j}(x)}\right) \leqq n+1$ for every $j$. For every $\alpha \in A_{m_{j+1}(x)}$ let

23) This mesh is one with respect to an arbitrary but fixed metric on $R$ agreeing with the preasigned topology of $R$. 
(7.14) $V_{\alpha}$ be the maximal open set with $\operatorname{Star}\left(V_{\alpha}, \mathfrak{H}_{m_{j+1}(x)}\right) \subset U_{\alpha}$ and

(7.15) $\mathfrak{B}_{m_{j}(x)}=\left\{V_{\alpha} ; \alpha \in A_{m_{j+1}(x)}\right\}$.

Since $\mathfrak{H}_{m_{j(x)}}>\mathfrak{H}_{m_{j+1}(x)}^{*}$, it holds that $\mathfrak{U}_{m_{j}(x)}>\mathfrak{B}_{m_{j}(x)}>\mathfrak{H}_{m_{j+1}(x)}$ and hence $\mathfrak{B}_{m_{j}(x)}$ is an open covering of $R$. Let $U_{\beta}$ be an arbitrary element of $\mathfrak{u}_{m_{j+1}(x)}$ with $x \in U_{\beta}$. When $U_{\beta} \cap V_{\alpha} \neq \phi$ where $V_{\alpha} \in \mathfrak{V}_{m_{j}(x)}, U_{\alpha}$ contains $x$. Therefore a number of elements of $\mathfrak{B}_{m_{j}(x)}$ which meet $U_{\beta}$ is at most $n+1$ by the inequality order $\left(x, \mathfrak{u}_{m_{j}(x)}\right) \leqq$ $n+1$. Thus $\mathfrak{B}_{m_{j}(x)}$ is locally finite at $x$ and $\operatorname{order}\left(x, \mathfrak{V}_{m_{j}(x)}\right) \leqq n+1, j=1,2, \cdots$. Take $m_{j}(x)$ with $m_{j}(x) \geqq i$ as well as $j \geqq 2$. Let $\alpha$ be an arbitrary element of $A_{m_{j}(x)}$. Since $F_{\alpha} \subset \bigcup\left\{U_{\beta} ; \beta \in A_{m_{j}(x)}, f_{m_{j+1}(x)}, m_{j}(x)(\beta)=\alpha\right\} \subset \bigcup\left\{U_{\beta} ; \beta \in A_{m_{j+1}(x)}\right.$, $\left.\operatorname{Star}\left(U_{\beta}, \mathfrak{H}_{m_{j+1}(x)}\right) \subset U_{\alpha}\right\} \subset V_{\alpha}$ and $\mathfrak{V}_{m_{j-1}(x)}=\left\{V_{\alpha} ; \alpha \in A_{m_{j}(x)}\right\}$ is locally finite at $x$, $\widetilde{F}_{m_{j}(x)}=\left\{F_{\alpha} ; \alpha \in A_{m_{j}(x)}\right\}$ is locally finite at $x$. Let $F_{\gamma}$ be an arbitrary element of $\widetilde{\mho}_{i}$ and then $F_{\gamma}=\bigcup\left\{F_{\beta} ; f_{m_{j}(x), i}(\beta)=\gamma\right\}$. Hence $\widetilde{F}_{i}$ is locally finite at $x$. Since $x$ was an arbitrary point of $R, \widetilde{F}_{i}$ is locally finite at every point of $R$.

Next we shall prove that each element of $\mathfrak{F}_{i}$ is a closed subset of $R$. Since $\bar{\mho}_{j}$ is locally finite and $F_{\alpha} \subset V_{\alpha} \subset \bar{V}_{\alpha} \subset U_{\alpha}$ for every $\alpha \in A_{j}$, there exists a locally finite open covering $\mathcal{G}_{j}=\left\{G_{\alpha} ; \alpha \in A_{j}\right\}$ of $R$ such that $\bar{F}_{\alpha} \subset G_{\alpha} \subset U_{\alpha}$ for every $\alpha \in A_{j}$ by [15, Lemma on pape 22]. Let $\alpha_{i}$ be an arbitrary element of $A_{i}$. Since $\bar{F}_{\alpha_{i}}=\bigcup\left\{\bar{F}_{\alpha_{i+j}} ; f_{i+j, i}\left(\alpha_{i+j}\right)=\alpha_{i}\right\}$ for any positive integer $j, \bar{F}_{\alpha_{i}} \subset \bigcap_{j=1}^{\infty}\left(\bigcup\left\{G_{\alpha_{i+j}}\right.\right.$; $\left.\left.f_{i+j, i}\left(\alpha_{i+j}\right)=\alpha_{i}\right\}\right) \subset \bigcap_{j=1}^{\infty}\left(\bigcup\left\{U_{\alpha_{i+j}} ; f_{i+j, i}\left(\alpha_{i+j}\right)=\alpha_{i}\right\}\right)=F_{\alpha_{i}}$. Thus $\widetilde{\mho}_{i}$ is a locally finite closed covering of $R$. Evidently a sequence $\mathfrak{F}_{i}, i=1,2, \cdots$, satisfies the conditions (7.2), (7.3) and (7.4). Therefore we get $\operatorname{dim} R \leqq n$ by Theorem 7.2. Thus the theorem is completely proved.

As a direct consequence of this theorem we get the following theorem.

Corollary 7.6 (J. Nagata [24, Theorem 3]). In order that a topological space $R$ be a metrizable space with $\operatorname{dim} R \leqq n$ it is necessary and sufficient that there exists a sequence of open coverings $\mathfrak{H}_{i}, i=1,2, \cdots$, of $R$ which satisfies the following conditions.

(7.16) $\mathfrak{U}_{i+1}^{*}$ refines $\mathfrak{U}_{i}$ for every $i$.

(7.17) order $\mathfrak{H}_{i} \leqq n+1$ for every $i$.

(7.18) For any point $x$ of $R$ and any neighborhood $U$ of $x$ there exists $i$ with $\operatorname{Star}\left(x, \mathfrak{H}_{i}\right) \subset U$.

\section{$\S 8$. Subordinate metric spaces}

Let $R$ be a normal space and $\mathfrak{H}_{i}, i=1,2, \cdots$, a sequence of open coverings of $R$ such that $\mathfrak{U}_{1}>\mathfrak{H}_{2}^{*}>\mathfrak{U}_{2}>\mathfrak{U}_{3}^{*}>\cdots$. For any pair of points $x, y$ of $R$ define a non-negative function $\mathrm{D}(x, y)$ as follows (cf. J. W. Tukey [29, Chap. 5]).

(8.1) When $x \notin \operatorname{Star}\left(y, \mathfrak{U}_{1}\right), \mathrm{D}(x, y)=1$.

(8.2) When there exists $i$ such that $x \in \operatorname{Star}\left(y, \mathfrak{H}_{i}\right)$ and $x \notin \operatorname{Star}\left(y, \mathfrak{H}_{i+1}\right), \mathrm{D}(x, y)=$

(8.3) When $x \in \operatorname{Star}\left(y, \mathfrak{u}_{i}\right)$ for every $i, \mathrm{D}(x, y)=0$.

Then $\mathrm{D}$ satisfies the following conditions for any points $x, y, z \in R$.

(8.4) $\quad \mathrm{D}(x, x)=0$.

(8.5) $\mathrm{D}(x, y)=\mathrm{D}(y, x) \geqq 0$.

(8.6) $\mathrm{D}(x, y) \leqq 2 \max (\mathrm{D}(x, z), \mathrm{D}(z, y))$.

(8.7) For any positive number $\varepsilon,\{y ; \mathrm{D}(x, y)<\varepsilon\}$ is an open set of $R$. In other words $\mathrm{D}$ is a so-called $\mathrm{D}$-function. 
With the aid of the function $\mathrm{D}$ define a function $r(x, y)$ for any pair of points $x, y$ of $R$ as follows (cf. A. H. Frink [7]).

(8.8) $\quad r(x, y)=\inf \sum_{i=0}^{m} \mathrm{D}\left(x_{i}, x_{i+1}\right)$, where $x_{0}=x, x_{m+1}=y$ and the infimum is taken over all $m \geqq 0$ and sets $x_{1}, \cdots, x_{m}$ of points of $R$.

Then the inequalities $(1 / 4) \mathrm{D}(x, y) \leqq r(x, y) \leqq \mathrm{D}(x, y)$ hold for any pair of points $x, y$, of $R$. Moreover $r$ satisfies the following conditions for any points $x, y, z$ of $R$.

(8.9) $\quad r(x, x)=0$.

(8.10) $\quad r(x, y)=r(y, x) \geqq 0$.

(8.11) $r(x, y) \leqq r(x, z)+r(z, y)$.

(8.12) For any positive number $\varepsilon,\{y ; r(x, y)<\varepsilon\}$ is an open set of $R$.

In other words $r$ is a pseudo-metric on $R$.

Let $F_{x}=\{y ; r(x, y)=0\}$; then $F_{x}$ is a closed set of $R$. Evidently for any point $y \in F_{x}$ we get $F_{x}=F_{y}$. Thus $R$ can be decomposed into a sum of mutually disjoint closed sets $\left\{F_{x} ; x \in R\right\}$. Let $R^{*}=\left\{x^{*}\right\}$ be a set of points $x^{*}$ such that there is a one-to-one correspondence: $F_{x} \leftrightarrow x^{*}$.

Let

(8.13) $r^{*}\left(x^{*}, y^{*}\right)=r\left(x^{\prime}, y^{\prime}\right)$, where $x^{\prime} \in F_{x}$ and $y^{\prime} \in F_{y}$;

then $r^{*}$ is independent of the special choice of $x^{\prime} \in F_{x}$ and $y^{\prime} \in F_{y}$ and hence uniquely defined. $r^{*}$ satisfies the following conditions for any points $x^{*}, y^{*}, z^{*}$ of $R^{*}$.

(8.14) $r^{*}\left(x^{*}, y^{*}\right)=0$ if and only if $x^{*}=y^{*}$.

(8.15) $r^{*}\left(x^{*}, y^{*}\right)=r^{*}\left(y^{*}, x^{*}\right) \geqq 0$.

(8.16) $\quad r^{*}\left(x^{*}, y^{*}\right) \leqq r^{*}\left(x^{*}, z^{*}\right)+r^{*}\left(z^{*}, y^{*}\right)$.

Thus $R^{*}$ is a metric space with the metric $r^{*}$. Define a mapping $\varphi: R \rightarrow R^{*}$ as $\varphi(x)=x^{*}$. Then $\varphi$ is continuous.

Definition 8.1. $\left(R^{*}, r^{*}\right)$ is called the subordinate metric space of $R$ with respect to a (normal) sequence $\mathfrak{H}_{i}, i=1,2, \cdots$.

Theorem 8.2. Let $R$ be a normal space and $\mathfrak{U}_{1}>\mathfrak{U}_{2}{ }^{*}>\mathfrak{H}_{2}>\mathfrak{H}_{3} *>\cdots$, be a normal sequence of open coverings of $R$ which satisfies the following conditions. ${ }^{24)}$ (8.17) Any element of any $\mathfrak{H}_{i+1}$ intersects at most a finite number of elements of $\mathfrak{U}_{i}$.

(8.18) order $\mathfrak{H}_{i} \leqq n+1$ for every $i$.

Let $\left(R^{*}, r^{*}\right)$ be the subordinate metric space of $R$ with respect to $\mathfrak{H}_{i}, i=1,2, \cdots$. Then we get $\operatorname{dim} R^{*} \leqq n$.

Proof. Let $\mathfrak{H}_{i}=\left\{U_{\alpha} ; \alpha \in A_{i}\right\}$ and

(8.19) $\mathfrak{V}_{i}=\left\{U_{\alpha}^{*}=\left\{x^{*} ; F_{x} \subset U_{\alpha}\right\} ; \alpha \in A_{i}\right\}$.

Then

(8.20) order $\mathfrak{B}_{i} \leqq$ order $\mathfrak{U}_{i} \leqq n+1$ for every $i$.

To prove that $\mathfrak{B}_{i}$ is a covering of $R^{*}$ let $x^{*}$ be an arbitrary point of $R^{*}$ and $y$ be an arbitrary point of $F_{x}$. Take $U_{a} \in \mathfrak{H}_{i}$ such that $\operatorname{Star}\left(y, \mathfrak{H}_{i+1}\right) \subset U_{\alpha}$. Let $z$ be an arbitrary point of $R-U_{\alpha}$ and then $z \notin \operatorname{Star}\left(y, \mathfrak{u}_{i+1}\right)$ and hence $\mathrm{D}(y, z) \geqq 2^{-i}$. Therefore $r(y, z) \geqq 2^{-i-2}$ and $z \notin F_{y}=F_{x}$, which proves $F_{x} \subset U_{a}$ and hence $x^{*} \in U_{a}^{*}$.

24) It is to be noted that when $R$ is a normal space with $\operatorname{dim} R \leqq n$, there is for any normal open covering (s) of $R$ a normal sequence which satisfies the conditions of the proposition such that $\mathfrak{u}_{1}<\mathbb{B}$. 
Thus $\mathfrak{B}_{i}$ covers $R^{*}$.

Next let us prove that

(8.21) $\overline{\mathfrak{B}}_{i+1}$ refines $\mathfrak{B}_{i}$.

Let $U_{\alpha}{ }^{*}$ be an arbitrary element of $\mathfrak{B}_{i+1}$ and $U_{\beta}$ an element of $\mathfrak{H}_{i}$ such that $\operatorname{Star}\left(U_{\alpha}, \mathfrak{H}_{i+1}\right) \subset U_{\beta}$. When $U_{\beta}{ }^{*}=R^{*}$, it evidently holds that $\overline{U_{\alpha}{ }^{*}} \subset U_{\beta}{ }^{*}$. Consider the case of $U_{\beta}{ }^{*} \neq R^{*}$. Let $y^{*}$ be an arbitrary point of $R^{*}-U_{\beta}{ }^{*}$; then $F_{y}-U_{\beta}$ is not empty. Hence there exists $x \notin U_{\beta}$ with $y^{*}=x^{*}$. Evidently $\operatorname{Star}\left(x, \mathfrak{u}_{i+1}\right) \cap U_{\alpha}=$ $\phi$. Let $z$ be an arbitrary point of $\left\{z ; r(x, z)<2^{-i-2}\right\}$; then $\mathrm{D}(x, z)<2^{-i}$ by the inequality $(1 / 4) \mathrm{D}(x, z) \leqq r(x, z)$. Hence $z \in \operatorname{Star}\left(x, \mathfrak{H}_{i+1}\right)$ and we get $\{z ; r(x, z)<$ $\left.2^{-i-2}\right\} \cap U_{\alpha}=\phi$. Therefore $\left\{z^{*} ; r^{*}\left(x^{*}, z^{*}\right)<2^{-i-2}\right\} \cap U_{\alpha^{*}}=\phi$, which proves $x^{*}=$ $y^{*} \notin \overline{U_{\alpha}{ }^{*}}$. Thus we can conclude that $\overline{U_{\alpha}{ }^{*}} \subset U_{\beta}{ }^{*}$, which proves (8.21).

Now we proceed to prove that $\mathfrak{B}_{i}$ is locally finite. Let $x^{*}$ be an arbitrary point of $R^{*}$ and $U_{\alpha}$ an element of $\mathfrak{u}_{i+1}$ such that $\operatorname{Star}\left(x, \mathfrak{U}_{i+2}\right) \subset U_{\alpha}$. Let $y$ be an arbitrary point of $\left\{y ; r(x, y)<2^{-i-3}\right\}$ and then $\mathrm{D}(x, y) \leqq 4 r(x, y)<2^{-i-1}$. Hence $y \in \operatorname{Star}\left(x, \mathfrak{H}_{i+2}\right)$. If $r(y, z)=0, r(x, z) \leqq r(x, y)+r(y, z)=r(x, y)$ and hence $z \in \operatorname{Star}\left(x, \mathfrak{U}_{i+2}\right)$. Thus $F_{y} \subset U_{x}$. Therefore $U\left(x^{*}\right)=\left\{y^{*} ; r^{*}\left(x^{*}, y^{*}\right)<2^{-i-3}\right\} \subset U_{a}{ }^{*}$. Since $U_{\alpha}$ intersects at most a finite number of elements of $\mathfrak{U}_{i}, U_{\alpha}{ }^{*}$ intersects at most a finite number of elements of $\mathfrak{B}_{i}$. Therefore $U\left(x^{*}\right)$ is an open neighborhood of $x^{*}$ which intersects at most a finite number of elements of $\mathfrak{B}_{i}$, which proves the local finiteness of $\mathfrak{B}_{i}$.

Finally let us prove that a sequence $\mathfrak{B}_{i}, i=1,2, \cdots$, satisfies the following condition.

(8.22) For any neighborhood $U^{*}$ of any point $x^{*}$ of $R^{*}$ there exists $i$ such that $\operatorname{Star}\left(x^{*}, \mathfrak{B}_{i}\right) \subset U^{*}$.

Let $i$ be a positive integer such that $S^{*}=\left\{y^{*} ; r^{*}\left(x^{*}, y^{*}\right)<2^{-i+1}\right\} \subset U^{*}$. Let $S=$ $\left\{y ; r(x, y)<2^{-i+1}\right\}$ and then $\varphi^{-1}\left(S^{*}\right)=S$. Since $r(x, y) \leqq \mathrm{D}(x, y),\left\{y ; \mathrm{D}(x, y)<2^{-i+1}\right\} \subset S$ and hence $\operatorname{Star}\left(x, \mathfrak{U}_{i}\right) \subset S$. If $x^{*} \in U_{\alpha^{*}} \in \mathfrak{B}_{i}, F_{x} \subset U_{\infty} \in \mathfrak{H}_{i}$ and hence $x \in U_{a} \in \mathfrak{H}_{i}$. Thus $U_{\alpha} \subset \operatorname{Star}\left(x, \mathfrak{H}_{i}\right)$. Hence $\varphi^{-1}\left(\operatorname{Star}\left(x^{*}, \mathfrak{B}_{i}\right)\right) \subset \operatorname{Star}\left(x, \mathfrak{H}_{i}\right)$ by $\varphi^{-1}\left(U_{\alpha}^{*}\right) \subset U_{\alpha}$. Therefore $U^{*} \supset S^{*}=\varphi(S) \supset \varphi\left(\operatorname{Star}\left(x, \mathfrak{H}_{i}\right)\right) \supset \operatorname{Star}\left(x^{*}, \mathfrak{B}_{i}\right)$.

At last we can conclude by Theorem 7.2 that $\operatorname{dim} R^{*} \leqq n$ and the proof is completed.

\section{\$9. Dimension-preserving completion}

Let $R$ be a metrizable space with $\operatorname{dim} R \leqq n$. Let $\mathfrak{U}_{1}$ be a locally finite open covering of $R$ such that order $\overline{\mathfrak{H}_{1}} \leqq n+1$ and mesh $\mathfrak{U}_{1}<2^{-1}$. Let $\mathfrak{H}_{2}$ be a locally finite open covering of $R$ such that i) order $\overline{\mathfrak{H}_{2}} \leqq n+1$, ii) mesh $\mathfrak{H}_{2}<2^{-2}$, iii) $\mathfrak{U}_{2}{ }^{*}$ refines $\mathfrak{H}_{1}$ and iv) $\mathfrak{U}_{2}$ refines $\left\{U(x)=R-\bigcup\left\{\bar{U}_{\alpha} ; x \notin \bar{U}_{\alpha}, U_{\alpha} \in \mathfrak{H}_{1}\right\} ; x \in R\right\}$. Then each element of $\mathfrak{H}_{2}$ intersects at most $n+1$ elements of $\mathfrak{H}_{1}$. Proceeding this procedure we get a sequence of open coverings $\mathfrak{U}_{i}, i=1,2, \cdots$, such that ${ }^{25}$ )

(9.1). $\mathfrak{U}_{1}>\mathfrak{H}_{2} *>\mathfrak{H}_{2}>\mathfrak{H}_{3} *>\cdots$,

(9.2) for any neighborhood $U$ of any point $x$ of $R$ there exists $i$ with $\operatorname{Star}\left(x, \mathfrak{U}_{i}\right) \subset$ $U$,

(9.3) each element of $\mathfrak{U}_{i+1}$ intersects at most $n+1$ elements of $\mathfrak{u}_{i}$.

Theorem 9.1. Let $R$ be a metrizable space with $\operatorname{dim} R \leqq n$. Let $\mathfrak{U}_{i}=$

25) The existence of the sequence of this property was for the first time proved by J. Nagata [24, Theorem 2]. 
$\left\{U_{\alpha} ; \alpha \in A_{i}\right\}, i=1,2, \cdots$, be a sequence of open coverings of $R$ satisfying the conditions (9.1), (9.2) and (9.3). Let $S$ be a completion of $R$ with respect to $\left\{\mathfrak{H}_{i} ; i=\right.$ $1,2, \cdots\}$. Then we get $\operatorname{dim} S \leqq n$.

Proof. For any open set $G$ of $R$ let $G^{*}=S-($ the closure of $R-G$ in $S){ }_{.}^{26)}$ Then it holds by Morita [31, Theorems 3, 4 and Lemma 7] that $\mathfrak{B}_{i}=\left\{U_{\alpha^{*}} ; \alpha \in A_{i}\right\}$, $i=1,2, \cdots$, satisfy the following conditions.

(9.4) $\left\{\mathfrak{V}_{i} ; i=1,2, \cdots\right\}$ gives a uniformity of $S$ which agrees with the topology of $S$.

(9.5) $\mathfrak{B}_{i+1}$ is a star-refinement of $\mathfrak{B}_{i}, i=1,2, \cdots$.

(9.6) Each element of $\mathfrak{V}_{i+1}$ meets at most $n+1$ elements of $\mathfrak{V}_{i}$.

Hence by Nagata [24, Theorem 2] or Theorem 7.5 we get $\operatorname{dim} S \leqq n$.

Remark 9.2. Another expression of Theorem 9.1 is as follows: Let $R$ be a metrizable space with $\operatorname{dim} R \leqq n$. Let $\mathfrak{H}_{i}, i=1,2, \cdots$, be a sequence of open coverings of $R$ which satisfies the conditions (9.1), (9.2) and (9.3). Let $r$ be a metric on $R$ defined in $\S 8$ with the usage of $\mathfrak{H}_{i}, i=1,2, \cdots$. Then a completion $S$ of $R$ with respect to $r$ has dimension $\leqq n$.

Definition 9.3. Recently J. Nagata [24, Theorem 5] has proved the following theorem: In order that $\operatorname{dim} R \leqq n$ for a metrizable space $R$ it is necessary and sufficient that we can define a metric $\rho(x, y)$ agreeing with the topology of $R$ such that for every $\varepsilon>0$ and for every point $x$ of $R, \rho\left(S_{\varepsilon / 2}(x), y_{i}\right)<\varepsilon(i=1, \cdots$, $n+2)$ imply $\rho\left(y_{i}, y_{j}\right)<\varepsilon$ for some $i, j$ with $i \neq j$, where $S_{\varepsilon / 2}(x)=\{y ; \rho(x, y)<\varepsilon / 2\}$. We call a metric $\rho$ with this property J. Nagata's metric.

Theorem 9.4. Let $R$ be a metrizable space with $\operatorname{dim} R \leqq n$. Then a completion $S$ of $R$ with respect to $J$. Nagata's metric $\rho$ on $R$ has dimension $\leqq n$.

Proof. Let $M_{1}$ be a maximal subset of $R$ such that $x, y \in M_{1}$ and $x \neq y$ imply $\rho(x, y) \geqq 1 / 2$. By the maximal property of $M_{1} \mathfrak{B}_{1}=\left\{S_{1 / 2}(x) ; x \in M_{1}\right\}$ is evidently an open covering of $R$. Let $\mathfrak{H}_{1}=\left\{\bigcup\left\{S_{1 / 2^{3}}(y) ; y \in S_{1 / 2}(x)\right\} ; x \in M_{1}\right\}$; then $\mathfrak{H}_{1}$ is also an open covering of $R$. Let $x$ be an arbitrary point of $R$. If $S_{1 / 2^{3}}(x) \cap$ $\left(\bigcup\left\{S_{1 / 2}{ }^{3}(y) ; y \in S_{1 / 2}\left(x_{i}\right)\right\}\right) \neq \phi, \quad i=1, \cdots, n+2$, for $x_{i} \in M_{1}$, then $\rho\left(S_{1 / 2}(x), x_{i}\right)<1 / 2$. Hence $\rho\left(x_{i}, x_{j}\right)<1 / 2$ for some $x_{i}$ and $x_{j}$. This implies $x_{i}=x_{j}$. by the property of $M_{1}$. Therefore $S_{1 / 2^{3}}(x)$ for an arbitrary point $x$ of $R$ intersects at most $n+1$ sets of $\mathfrak{H}_{1}$. Let $M_{2}$ be a maximal subset of $R$ such that $x, y \in M_{2}$ and $x \neq y$ imply $\rho(x, y) \geqq 1 / 2^{6}$. By the maximal property of $M_{2} \mathfrak{B}_{2}=\left\{S_{1 / 2^{6}}(x) ; x \in M_{2}\right\}$ is an open covering of $R$. Let $\mathfrak{H}_{2}=\left\{\bigcup\left\{S_{1 / 2^{8}}(y) ; y \in S_{1 / 2^{6}}(x)\right\} ; x \in M_{2}\right\}$; then $\mathfrak{H}_{2}$ is an open covering of $R$. Since $\mathfrak{H}_{1}>\left\{S_{1 / 2^{3}}(x) ; x \in R\right\}>\left\{S_{1 / 2^{5}}(x) ; x \in R\right\}^{*}>\left\{S_{1 / 2}(x) ; x \in R\right\}>\mathfrak{H}_{2}$, $\mathfrak{H}_{2}{ }^{*}$ refines $\mathfrak{H}_{1}$ and each element of $\mathfrak{H}_{2}$ intersects at most $n+1$ elements of $\mathfrak{U}_{1}$.

In general let $M_{i}$ be a maximal subset of $R$ such that $x, y \in M_{i}$ and $x \neq y$ imply $\rho(x, y) \geqq 2^{-1-5(i-1)}$. Then $\mathfrak{B}_{i}=\left\{S_{2-1-5(i-1)}(x) ; x \in M_{i}\right\}$ is an open covering of $R$. Let $\mathfrak{H}_{i}=\left\{\bigcup\left\{S_{2-3-5(i-1)}(y) ; y \in S_{2^{-1-5(i-1)}}(x)\right\} ; x \in M_{i}\right\}$; then by the same argument as in the above $\mathfrak{U}_{i}{ }^{*}$ refines $\mathfrak{U}_{i-1}$ and each element of $\mathfrak{U}_{i}$ intersects at most $n+1$ elements of $\mathfrak{H}_{i-1}, i=2,3, \cdots$. It is to be noted that the identity mapping of $R$ onto $R$ is a homeomorphism of $\left\{R, \mathfrak{H}_{i}(i=1,2, \cdots)\right\}$ onto $\{R, \rho\}$.

Since mesh $\mathfrak{U}_{i}$ with respect to $\rho$ converges to 0 as $i \rightarrow \infty$, every Cauchy

26) cf. Morita [32, Lemma 1]. 
sequence of $\left\{R, \mathfrak{U}_{i}(i=1,2, \cdots)\right\}$ is a Cauchy sequence with respect to $\rho$.

Conversely let us show that every Cauchy sequence with respect to $\rho$ is a Cauchy sequence of $\left\{R, \mathfrak{U}_{i}(i=1,2, \cdots)\right\}$. Let $\left\{x_{i}\right\}=\left\{x_{i} ; i=1,2, \cdots\right\}$ be an arbitrary Cauchy sequence of $\{R, \rho\}$ and $j$ an arbitrary positive integer; then there exists $k$ such that for any $m \geqq k x_{m} \in S_{2-3-5(j-1)}\left(x_{k}\right)$. Since $\mathfrak{B}_{j}$ covers $R$, there exists a point $x_{0} \in M_{i}$ such that $x_{k} \in S_{2-1-5(j-1)}\left(x_{0}\right)$. Since $S_{2-3-5(j-1)}\left(x_{k}\right) \subset$ $\bigcup\left\{S_{2-3-5(j-1)}(y) ; y \in S_{2-1-5(j-1)}\left(x_{0}\right)\right\} \in \mathfrak{U}_{j}$, we get $S_{2-3-5(j-1)}\left(x_{k}\right) \subset \operatorname{Star}\left(x_{k}, \mathfrak{U}_{j}\right)$. Therefore $x_{m} \in \operatorname{Star}\left(x_{k}, \mathfrak{H}_{j}\right)$ for any $m \geqq k$, which proves that $\left\{x_{i}\right\}$ is a Cauchy sequence of $\left\{R, \mathfrak{H}_{i}(i=1,2, \cdots)\right\}$. Thus we can conclude that a completion $S$ of $\{R, \rho\}$ is essentially the same as a completion $S_{1}$ of $\left\{R, \mathfrak{H}_{i}(i=1,2, \cdots)\right\}$. Since a sequence of open coverings $\mathfrak{H}_{i}, i=1,2, \cdots$, of $R$ clearly satisfies the conditions (9.1), (9.2) and (9.3), we get $\operatorname{dim} S_{1} \leqq n$ by Theorem 9.1. Therefore we get $\operatorname{dim} S \leqq n$, which proves the theorem.

ReMark 9.5. The following is Morita's simple proof of Theorem 9.4 making no use of Theorem 9.1: Let $\bar{\rho}$ be a metric on $S$ generated by $\rho$ on $R$. Then it can be proved that $\bar{\rho}$ is Nagata's metric.

\section{$\S 10$. Length of coverings}

Definition 10.1. We call a covering $\mathfrak{H}$ of a space a multiplicative covering if every non-empty intersection $\bigcap_{i=1}^{k} U_{i}$ of elements $U_{i}, i=1, \cdots, k$, of $\mathfrak{u}$ is also an element of $\mathfrak{U}$. The maximal number $n$ such that there exists a sequence $U_{1} \supsetneq U_{2} \supsetneq \cdots \supsetneqq U_{n} \neq \phi$ of elements of a multiplicative covering $\mathfrak{U}$ is called the length of $\mathfrak{U}$, length $\mathfrak{U}$. We mean by the rank of an element $U$ of $\mathfrak{U}$ the maximal number $r$ such that there exists a sequence $U=U_{1} \supsetneqq U_{2} \supsetneqq \cdots \supsetneqq U_{r}$ of elements of $\mathfrak{H}$.

Under these terminologies J. Nagata's theorem [24, Theorem 4] is as follows: In order that a topological space $R$ be a metrizable space with $\operatorname{dim} R \leqq n$ it is necessary and sufficient that there exists a sequence $\mathfrak{U}_{1}>\mathfrak{H}_{2} *>\mathfrak{U}_{2}>\mathfrak{U}_{3} *>\cdots$ of multiplicative open coverings with length $\leqq n+1$ such that $\left\{\operatorname{Star}\left(p, \mathfrak{H}_{m}\right) ; m=\right.$ $1,2, \cdots\}$ is a neighborhood basis of $p$ for every point $p$ of $R$.

Theorem 10.2. In order that a topological space $R$ be a metrizable space with $\operatorname{dim} R \leqq n$ it is necessary and sufficient that there exists a sequence of coverings $\mathfrak{H}_{i}=\left\{H_{\infty} ; \alpha \in A_{i}\right\}, i=1,2, \cdots$, of $R$ which satisfies the following conditions.

(10.1) $\mathfrak{S}_{i}$ is a locally finite multiplicative covering for every $i$.

(10.2) $\overline{\mathfrak{S}}_{i+1}$ refines $\mathfrak{S}_{i}$ for every $i$.

(10.3) length $\mathfrak{S}_{i} \leqq n+1$ for every $i$.

(10.4) For any point $x$ of $R$ and any neighborhood $U$ of $x$ there exists $i$ with $\operatorname{Star}\left(x, \mathfrak{S}_{i}\right) \subset U$.

Proof. If $R$ is a metric space with $\operatorname{dim} R \leqq n$, there exists a sequence of locally finite coverings $\mathfrak{F}_{i}, i=1,2, \cdots$, of $R$ with the conditions (3.1), (3.2), (3.3). Since $\widetilde{\mho}_{i}$ plus all the non-empty intersections of a finite number of elements of $\mathfrak{\mho}_{i}$ is obviously a multiplicative covering with length $\leqq n+1$, the necessity of the theorem is valid.

Let us assume the existence of a sequence satisfying the conditions of the proposition. We can assume without loss of generality that if $\alpha$ and $\beta$ are 
elements of $A_{i}$ with $\alpha \neq \beta$, then $H_{\alpha} \neq H_{\beta}$. Since the conditions (10.2) and (10.4) yield the condition (7.1), $R$ is metrizable by Lemma 7.1.

We shall prove $\operatorname{dim} R \leqq n$ by the induction on $n$. When $n=0$, length $\mathfrak{H}_{i} \leqq 1$ implies order $\mathfrak{S}_{i} \leqq 1$. Moreover $\mathfrak{g}_{i}$ is a closed covering by (10.2). Hence we get $\operatorname{dim} R \leqq 0$ by Theorem 3.2. Now make the induction assumption that if there is a sequence which satisfies the conditions (10.1), (10.2), (10.4) and

(10.5) length $\mathfrak{S}_{i} \leqq n$ for every $i$,

then we get $\operatorname{dim} R \leqq n-1$.

Now let $\mathfrak{S}_{i}, i=1,2, \cdots$, be a sequence satisfying the conditions of the theorem. For every $i$ let us define a single-valued mapping $f_{i+1, i}: A_{i+1} \rightarrow A_{i}$ in such a way that $f_{i+1, i}\left(\alpha_{i+1}\right)=\alpha_{i}$ yields $\bar{H}_{\alpha_{i+1}} \subset H_{\alpha_{i}}$. For $i$ and $j$ with $i>j$ let $f_{i j}=f_{j+1, j} \cdots f_{i-1, i-2} f_{i, i-1}$. Let $A_{0}$ be the aggregate of points $a=\left(\alpha_{1}, \alpha_{2}, \cdots\right)$ of $\lim \left\{A_{i}, f_{i j}\right\}$, where every $A_{i}$ is a topological space with discrete topology, such that $\bigcap_{i=1}^{\infty} H_{\alpha_{i}} \neq \phi$, and $f$ a mapping of $A_{0}$ into $R$ defined by $f(a)=\bigcap_{i=1}^{\infty} H_{\pi_{i}(a)}$, where $\pi_{i}$ is the projection of $A_{0}$ into $A_{i}$. Then we can verify by the same argument as in the proof of $(\mathrm{B}) \rightarrow(\mathrm{C})$ of $\S 3$ that i) $f$ is onto and ii) $\mho_{i}=\left\{F_{\alpha}=f\left(\pi_{i}{ }^{-1}(\alpha)\right)\right.$; $\left.\alpha \in A_{i}\right\}$ is a locally finite closed covering of $R$ such that (10.6) for any $i$ and any $\alpha \in A_{i}, F_{\alpha}=\bigcup\left\{F_{\beta} ; f_{i+1, i}(\beta)=\alpha\right\}$.

Let

(10.7) $F_{i}=\left\{x ; x \in R\right.$, order $\left.\left(x, \widetilde{\mho}_{i}\right)>1\right\}, i=1,2, \cdots$;

then it can easily be seen that for every $i F_{i}$ is a closed subset of $R$. Let $x$ be an arbitrary point of $F_{i}$; then there exist $\alpha_{i}$ and $\beta_{i}$ of $A_{i}$ with $\alpha_{i} \neq \beta_{i}$ such that $x \in F_{\alpha_{i}} \cap F_{\beta_{i}}$. Then by (10.6) there exist $\alpha_{i+1}$ and $\beta_{i+1}$ with $f_{i+1, i}\left(\alpha_{i+1}\right)=\alpha_{i}$ and $f_{i+1, i}\left(\boldsymbol{\beta}_{i+1}\right)=\beta_{i}$ such that $x \in F_{\alpha_{i+1}} \cap F_{\beta_{i+1}}$. Hence $F_{i} \subset F_{i+1}$.

Moreover we can conclude from the above argument that if we set

(10.8) $\quad \mathfrak{S}_{j}{ }^{\prime}=\left\{H_{\alpha_{j}} \cap H_{\beta_{j}} ; f_{j i}\left(\alpha_{j}\right) \neq f_{j i}\left(\beta_{j}\right), F_{f_{j i}\left(\alpha_{j}\right)} \cap F_{f_{j i}\left(\beta_{j}\right)} \neq \phi\right\}, j=i, i+1, \cdots$, and

(10.9) $\mathfrak{S}_{j}{ }^{\prime \prime}=\mathfrak{H}_{j}{ }^{\prime}$ plus all the non-empty intersections of a finite number of elements of $\mathfrak{g}_{j}{ }^{\prime}, j=i, i+1, \cdots$,

then $F_{i} \cap \mathfrak{S}_{j}{ }^{\prime \prime}, j=i, i+1, \cdots$, is a sequence of locally finite multiplicative coverings of $F_{i}$ which satisfies the following conditions.

(10.10) $\overline{F_{i} \cap \mathfrak{S}_{j+1}^{\prime \prime}}=F_{i} \cap \overline{\mathfrak{S}_{j+1}^{\prime \prime}}$ refines $F_{i} \cap \mathfrak{S}_{j}^{\prime \prime}$ for $j=i, i+1, \cdots$.

(10.11) length $F_{i} \cap \mathfrak{S}_{j}{ }^{\prime \prime} \leqq n$ for $j=i, i+1, \cdots$.

(10.12) For any point $x$ of $F_{i}$ and any (relatively) open neighborhood $U$ of $x$ there exists $j(\geqq i)$ with $\operatorname{Star}\left(x, \overline{F_{i} \cap \mathfrak{S}_{j}^{\prime \prime}}\right) \subset U$.

Therefore we get

(10.13) $\operatorname{dim} F_{i} \leqq n-1, i=1,2, \cdots$,

by the induction assumption. Then by the sum theorem we get

(10.14) $\operatorname{dim} \bigcup_{i=1}^{\infty} F_{i} \leqq n-1$.

On the other hand $\left(R-F_{i}\right) \cap \mho_{i}$ is a locally finite closed covering of $R-F_{i}$ whose order $\leqq 1$. Hence

(10.15) $\quad \mathfrak{R}_{i}=\left(R-\bigcup_{j=1}^{\infty} F_{j}\right) \cap \mathfrak{F}_{i}$

is a locally finite closed covering of $R-\bigcup_{j=1}^{\infty} F_{j}$ whose order $\leqq 1$.

Thus a sequence $\mathfrak{R}_{i}, i=1,2, \cdots$, satisfies the following conditions.

(10.16) $\mathfrak{Q}_{i+1}$ refines $\mathfrak{Q}_{i}$ for every $i$.

(10.17) order $\mathfrak{L}_{i} \leqq 1$ for every $i$.

(10.18) For any $x \in R-\bigcup_{j=1}^{\infty} F_{j}$ and any neighborhood $U$ of $x$ there exists $i$ such that $\operatorname{Star}\left(x, \mathbb{R}_{i}\right) \subset U$.

Therefore by Theorem 3.2 we get 
(10.19) $\operatorname{dim}\left(R-\mathbf{U}_{j=1}^{\infty} F_{j}\right) \leqq 0$.

Combining (10.19) with (10.14) we get by Theorem $3.2 \operatorname{dim} R \leqq n$, which completes the induction. Thus the theorem is completely proved.

\section{$\S 11$. Characterization of dimension of non-metrizable spaces by means of length of coverings}

TheoRem 11.1. For a normal space $R$ the following conditions are equivalent. (11.1) $\operatorname{dim} R \leqq n$.

(11.2) Every finite open covering can be refined by a finite multiplicative open covering whose length $\leqq n+1$.

(11.3) Every locally finite open covering can be refined by a locally finite multiplicative open covering whose length $\leqq n+1$.

(11.4) Every finite open covering can be refined by a finite multiplicative closed covering whose length $\leqq n+1$.

(11.5) Every locally finite open covering can be refined by a locally finite multiplicative closed covering whose length $\leqq n+1$.

Proof. $\quad(11.1) \rightarrow(11.2)$ : Let $\operatorname{dim} R \leqq n$ and $\$$ an arbitrary finite open covering of $R$. Then there exists a finite open covering $\mathfrak{U}$ of $R$ with order $\mathfrak{U} \leqq n+1$ such that $\mathfrak{U}$ refines $\mathfrak{S}$. Let $\mathfrak{S}$ be $\mathfrak{U}$ plus all the non-empty intersections of a finite number of elements of $\mathfrak{U}$ and then it is obvious that $\mathfrak{S}$ is a finite multiplicative open covering of $R$ with length $\mathfrak{S} \leqq n+1$ such that $\mathfrak{S}$ refines $(\mathfrak{S}$.

The implications $(11.1) \rightarrow(11.3),(11.1) \rightarrow(11.4)$ and $(11.1) \rightarrow(11.5)$ are proved by the same way.

$(11.2) \rightarrow(11.1)$ is proved essentially by P. Alexandroff and A. Kolmogroff [1].

$(11.3) \rightarrow(11.1)$ : Let $\left(\mathbb{S}_{1}\right.$ be an arbitrary locally finite open covering of $R$ and $\mathfrak{S}_{1}$ be a locally finite multiplicative open covering of $R$ with length $\mathfrak{S}_{1} \leqq n+1$ and $\mathfrak{S}_{1}>\mathscr{H}_{1}$. Then by J. Dieudonné [2] there exists a locally finite open covering $\mathscr{B}_{2}$ of $R$ with $\mathscr{S}_{1}>\mathscr{S}_{2}{ }^{*}$. Let $\mathscr{S}_{2}$ be a locally finite multiplicative open covering of $R$ with length $\mathfrak{S}_{2} \leqq n+1$ and $\mathscr{S}_{2}>\mathfrak{S}_{2}$. Since $\left(\mathscr{G}_{1}>\mathfrak{S}_{1}>\mathscr{G}_{2} *>\mathfrak{S}_{2}>\mathfrak{S}_{2}\right.$, we get $\mathfrak{G}_{1}>\mathfrak{S}_{1}>\mathfrak{S}_{2}{ }^{*}$. Repeating this argument we get a sequence of locally finite multiplicative open coverings $\mathfrak{H}_{i}, i=1,2, \cdots$, of $R$ which satisfies the following conditions.

(11.6) $\quad \mathfrak{S}_{1}>\mathfrak{I}_{1}>\mathscr{S}_{2} *>\mathfrak{S}_{2}>\mathfrak{S}_{3} *>\cdots$

(11.7) length $\mathfrak{S}_{i} \leqq n+1$ for every $i$.

Then we can construct an open covering $\mathfrak{U}$ whose order $\leqq n+1$ such that $\mathfrak{U}$ refines $\left(\mathfrak{S}_{1}\right.$ by the same argument employed in J. Nagata [24, Theorem 4], which shows $\operatorname{dim} R \leqq n$.

$(11.5) \rightarrow(11.1)$ : Let $\$ \$ \xi=\left\{G_{\lambda} ; \lambda \in \Lambda\right\}$ be an arbitrary locally finite open covering of $R$ and $\mathfrak{S}=\left\{H_{\alpha} ; \alpha \in A\right\}$ a locally finite multiplicative closed covering of $R$ with length $\mathfrak{S} \leqq n+1$ and $\mathbb{S}>\mathfrak{S}$ such that whenever $\alpha \neq \beta(\alpha, \beta \in A), H_{\alpha} \neq H_{\beta}$. Let $\mathfrak{S}_{i}=\left\{H_{\alpha} ; \alpha \in A_{i}\right\}, \quad i=1,2, \cdots, n+1$, be the aggregate of elements of $\mathfrak{g}$ whose rank $=i$. Assume that $A=\{1,2, \cdots, \lambda, \cdots\}$ is well-ordered. Let $A_{i \lambda}=\left\{\alpha ; \alpha \in A_{i}\right.$,

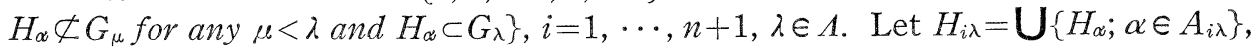
$i=1, \cdots, n+1, \lambda \in \Lambda$. Then $H_{i \lambda}$ is a closed set with $H_{1 \lambda} \subset G_{\lambda}$ for every $\lambda \in A$. Since order $\mathfrak{S}_{1} \leqq 1$, order $\left(H_{1 \lambda} ; \lambda \in \Lambda\right\} \leqq 1$. Hence there exists by Morita [15, Theorem 1.3] a collection of open sets $\mathfrak{H}_{1}=\left\{U_{1 \lambda} ; \lambda \in \Lambda\right\}$ with order $\mathfrak{H}_{1} \leqq 1$ such that 
$H_{1 \lambda} \subset U_{1 \lambda} \subset G_{\lambda}$ for every $\lambda \in \Lambda$.

It is to be noted that for any $\alpha, \beta \in A_{2}$ with $\alpha \neq \beta$ either $H_{\alpha} \cap H_{\beta} \in \mathfrak{S}_{1}$ or $H_{\infty} \cap H_{\beta}=\phi$ holds. Hence $\left.\left\{H_{2 \lambda}-\bigcup \bigcup U U_{1 \mu} ; \mu \in \Lambda\right\} ; \lambda \in A\right\}$ is a collection of mutually disjoint closed sets of $R$ such that $H_{2 \lambda}-\bigcup\left\{U_{1 \mu} ; \mu \in \Lambda\right\} \subset G_{\lambda}$ for every $\lambda \in \Lambda$. Therefore by Morita [15, Theorem 1.3] there exists a collection of open sets $\mathfrak{H}_{2}=\left\{U_{2 \lambda} ; \lambda \in \Lambda\right\}$ with order $\mathfrak{H}_{2} \leqq 1$ such that $H_{2 \lambda}-\bigcup\left\{U_{1 \mu} ; \mu \in \Lambda\right\} \subset U_{2 \lambda} \subset G_{\lambda}$ for every $\lambda \in \Lambda$.

Repeating such processes we get collections of open sets $\mathfrak{H}_{i}=\left\{U_{i \lambda} ; \lambda \in \Lambda\right\}$, $i=1,2, \cdots, n+1$, with order $\mathfrak{U}_{i} \leqq 1$ such that $H_{i \lambda}-\bigcup\left\{U_{j \mu} ; j=1, \cdots, i-1, \mu \in A\right\} \subset$ $U_{i \lambda} \subset G_{\lambda}$ fore every $\lambda \in A$. Since $\left\{H_{i \lambda} ; i=1, \cdots, n+1, \lambda \in A\right\}$ covers $R,\left\{U_{i \lambda} ; i=\right.$ $1, \cdots, n+1, \lambda \in \Lambda\}$ covers $R$. Therefore $\mathfrak{U}=\left\{U_{\lambda}=\bigcup\left\{U_{i \lambda} ; i=1, \cdots, n+1\right\} ; \lambda \in \Lambda\right\}$ is an open covering of $R$ such that $U_{\lambda} \subset G_{\lambda}$ for every $\lambda \in \Lambda$. Moreover it is evident that order $\mathfrak{U} \leqq n+1$. Thus an arbitrary locally finite open covering $\&$ can be refined by an open covering whose order $\leqq n+1$, which shows $\operatorname{dim} R \leqq n$.

The implication (11.4) $\rightarrow(11.1)$ is proved by the same way as above and the theorem is completely proved.

\section{Department of Mathematics Ehime University}

\section{References}

[1] P. Alexandroff and A. Kolmogoroff: Endliche Überdeckungen topologischer Räume, Fund. Math., 26 (1936), 267-271.

[2] J. Dieudonné: Une généralisation des espaces compacts, J. Math. Pures Appl., 23 (1944), 65-76.

[3] C. H. Dowker: Mapping theorems for non-compact spaces, Amer. J. Math., 69 (1947), 200-242.

[4] — Inductive dimension of completely normal spaces, Quart. J. Math., Oxford (2), 4 (1953), 267-281.

[5] - Local dimension of normal spaces, Quart. J. Math., Oxford (2), 6 (1955), 101-120.

[6] and W. Hurewicz: Dimension of metric spaces, Fund. Math., 43 (1956), 8388.

[7] A. H. Frink: Distance function and the metrization problem, Bull. Amer. Math. Soc., 44 (1937), 133-142.

[8] W. Hurewicz and H. Wallman: Dimension theory, 1941.

[9] W. Hurewicz: Über stetige Bilder von Punktmengen, Proc. Amsterdam Acad., 30 (1927), 164 .

[10] M. Katětov: On the dimension of non-separable spaces I, Czechoslovak Math. J., 2 (77) (1952), 333-368.

[11] — : On the dimension of non-separable spaces II, Czechoslovak Math. J., 6 (81) (1956), 485-516.

[12] — : On the relation between the metric and topological dimension, Czechoslovak Math. J., 8 (83) (1958), 163-166.

[13] S. Lefschetz: Algebraic topology, 1942.

[14] K. Morita: On the dimension of normal spaces I, Jap. J. Math., 20 (1950), 5-36.

[15] __ : On the dimension of normal spaces II, J. Math. Soc. Japan, 2 (1950), 16-33.

[16] — Normal families and dimension theory for metric spaces, Math. Ann., 128 (1954), 350-362.

[17] : On closed mappings and dimension, Proc. Japan Acad., 32 (1956), 161-165.

[18] - A condition for the metrizability of topological spaces and for $n$-dimensionality, Science Rep. Tokyo Kyoiku Daigaku, Sect. A, 5 (1955), 33-36. 
[19] K. Nagami: On the dimension of paracompact Hausdorff spaces, Nagoya Math. J., 8 (1955), 69-70.

[20] : Some theorems in dimension theory for non-separable spaces, J. Math. Soc. Japan, 9 (1957), 80-92.

[21] : ' Finite-to-one closed mappings and dimension I, Proc. Japan Acad., 34 (1958), 503-506.

[22] : Finite-to-one closed mappings and dimension II, Proc. Japan Acad., 35 (1959), 437-439.

[23] : Finite-to-one closed mappings and dimension III, Proc. Japan Acad., 36 (1960), 405-407.

[24] J. Nagata: Note on dimension theory for metric spaces, Fund. Math., 45 (1958), 143-181.

[25] - On a necessary and sufficient condition of metrizability, J. Inst. Polytech. Osaka City Univ., 1 (1950), Ser. A, 93-100.

[26] J. H. Roberts: A theorem on dimension, Duke Math. J., 8 (1941), 565-574.

[27] K. Sitnikov: An example of two-dimensional set in the three-dimensional euclidean space, which allows a deformation as small as desired in one-dimensional polyhedron, and some new character of the dimension of the sets in euclidean spaces, Doklady Acad. Nauk U.S.S.R., 88 (1953), 21-24.

[28] J. Suzuki: Note on a theorem for dimension, Proc. Japan Acad., 35 (1959), 201-202.

[29] J. W. Tukey: Convergence and uniformity in topology, 1940.

[30] N. Vedenisoff: Généralisation de quelques théorèmes sur la dimension, Comp. Math., 7 (1939), 194-200.

[31] K. Morita: On the simple extension of a space with respect to a uniformity I, Proc. Japan Acad., 27 (1951), 65-72.

[32] : On the simple extension of a space with respect to a uniformity II, Proc. Japan Acad., 27 (1951), 130-137. 